\title{
Computational and Numerical Investigation of the Batch Markovian Arrival Process Subject to Renewal Generated Geometric Catastrophes
}

\author{
Nitin Kumar ${ }^{1}$. Umesh Chandra Gupta ${ }^{1} \cdot$ Gagandeep Singh ${ }^{2,3}$ \\ Accepted: 16 July 2021 / Published online: 7 August 2021 \\ (c) The Author(s), under exclusive licence to Springer Nature India Private Limited 2021
}

\begin{abstract}
In this paper, we consider a stochastic model in which the population grows according to the batch Markovian arrival process and is subjected to renewal generated geometric catastrophes. Our analytical work starts from the vector generating function (VGF) of the population size at post-catastrophe epoch. We develop a methodology for extracting the population size distribution at post-catastrophe epoch from the VGF, which is based on the inversion of VGF using the roots method. The method is analytically quite simple and easy to implement. Further, we obtain the population size distribution at arbitrary, pre-catastrophe and prearrival epochs along with their factorial moments. To show the applicability and correctness of the proposed methodology, we match our results with the available ones in special cases and present several numerical examples for different inter-catastrophe time distributions. Moreover, we investigate the effect of key parameters on the system performance and display the results in the form of graphs along with a detailed description.
\end{abstract}

Keywords Batch Markovian arrival process · Geometric catastrophes · Population · Renewal process $\cdot$ Roots

\section{Introduction}

The occurrence of various forms of catastrophes, such as earthquake, epidemic, pandemic like Covid-19, etc, in an environment is very common and it usually results in a sudden decline

\footnotetext{
Umesh Chandra Gupta

umesh@maths.iitkgp.ac.in

Nitin Kumar

nitinkumar7276@gmail.com

Gagandeep Singh

rahigs@gmail.com

1 Department of Mathematics, Indian Institute of Technology, Kharagpur 721302, India

2 Centre of Advanced Study in Mathematics, Department of Mathematics, Panjab University, Chandigarh 160014, India

3 University Grants Commission, New Delhi, India
} 
in the population size. When the whole population becomes extinct after the occurrence of a catastrophe, it is considered as total catastrophe, see Economou and Fakinos [11], Economou [9]. However, when a catastrophe occurs and it does not destroy the whole population, rather some individuals survive then it is called mild annihilation phenomena or mild catastrophe, see Brockwell et al. [7], Economou [10], Economou and Gómez-Corral [12], Kumar and Gupta [19], Kumar and Gupta [20] and reference therein.

Economou and Gómez-Corral [12] analyzed a stochastic model in which population grows according to batch Markovian arrival process (BMAP), and catastrophes occur according to renewal generated process. When a catastrophe occurs, the individuals of the population begin to be annihilated sequentially, each with a fixed probability $p$. This sequential process is terminated at the first individual who survives with probability $q=1-p$ or when the whole population wipes out. They derived the vector generating function (VGF) of the population size distribution at post-catastrophe epoch using the embedded Markov chain technique, and proposed matrix-analytic method (MAM) to compute the equilibrium distribution of the population size which begins with the computation of $\mathbf{R}$ and $\mathbf{G}$ matrices. However, they did not present any numerical results.

As the numerical aspects of a mathematical model are as important as its theoretical development, there is the need to develop an alternative procedure that can be easily understood by the practitioners working in related areas due to limited understanding of the complex mathematical steps involved at the implementation stage. Moreover, the relevance of the model cannot be appreciated unless the detailed impact of model parameters on various performance measures is investigated.

In this paper, we propose an alternative procedure for extracting the population size distribution from the VGF. The proposed methodology is based on the inversion of VGF using the roots method and has been successfully used in recent years for the analysis of several queueing models with MAP/BMAP arrival processes, see e.g., Chaudhry et al. [8], Singh et al. [26], Singh et al. [28], Gupta et al. [14], Pradhan and Gupta [23], Bank and Samanta [4]. The method is analytically quite simple and easy to implement. It is shown in Gupta et al. [14], Singh et al. [28], Bank and Samanta [4] that the computation time using the roots method for different values of the parameters remains almost the same, whereas the MAM requires more computation time and is dependent on the parameter like lag-correlation and traffic intensity.

Besides obtaining the population size distribution at post-catastrophe and arbitrary epochs, we also obtain population size distributions at pre-catastrophe and pre-arrival epochs, which was missing in Economou and Gómez-Corral [12]. Further, we also present explicit expression of factorial moments of the population size at various epochs which have been directly derived from the VGF. Several numerical results have been presented in order to demonstrate our methodology. The impact of key parameters, including correlation, on the mean and second moment of population size has been critically examined.

It may be noted that in recent years a considerable amount of work has been carried out related to continuous as well as discrete-time population models with geometric catastrophes, see e.g., Barbhuiya et al. [5], Kumar et al. [21], Kumar and Gupta [18,20] and reference therein. In Barbhuiya et al. [5] and Kumar et al. [21], the authors investigate a geometric catastrophe population model in which population grows as per the batch renewal arrival process. Whereas in Kumar and Gupta [18] the analysis of a population model with geometric catastrophe has been carried out with the assumption that catastrophes occur according to the MAP and individuals arrive to the system as per the BMAP. They have obtained the VGF of the population size distribution at various epochs and presented a detailed computing process using roots methods to extract the distributions. In this sequence, Kumar and Gupta 
[20] investigated a discrete-time population model where individuals take place as per the D-MAP and catastrophes occur according to the renewal process. They have obtained the VGF of the population size distribution at various epochs as well as the factorial moments of the population size. To extract the distribution from the VGF, a detailed computing process using roots method is presented. For literature related to binomial catastrophes see, Kumar and Gupta [19], Economou [10], Brockwell et al. [7] and for total catastrophes see, Gupta et al. [15], Kumar et al. [22], and reference therein. One can refer to Shaikh et al. [24], Ahmad et al. [1], Ahmed et al. [2], Ghaffar et al. [13], Jhangeer et al. [16], Ali et al. [3], Khalid et al. [17], for recent work related to mathematical modeling, numerical and analytical methods.

The rest of the paper is organized as follows. In the upcoming section, we give a brief description of the model. In Sect. 3, we obtain the population size distribution at various epochs and present a detailed computing process for evaluation of the population size distribution at various epochs in Sect. 4. Further, in Sect. 5, we formulate the factorial moments of the population size at each epoch. We discuss some special cases of the model in Sect. 6. A few numerical results in the form of tables and graphs are given in Sect. 7 which is followed by the conclusion.

\section{Model Description and Notation}

Though the model is well described in Economou and Gómez-Corral [12]. However, for the sake of completeness we describe the model again:

- We assume that arrivals occur according to BMAP with representation $\left\{\mathbf{D}_{k} ; k=0,1, \ldots\right\}$ where $\mathbf{D}_{k}$ 's are square matrices of order $m$. The $(i, j)$ th element of $\mathbf{D}_{k}$, i.e., $\left(\mathbf{D}_{k}\right)_{i, j}$, is a transition rate from state $i$ to state $j$ with an arrival of batch having size $k, k \geq 1$. While the $(i, j)$ th element of $\mathbf{D}_{0}$, i.e., $\left(\mathbf{D}_{0}\right)_{i, j}$, is a transition rate from state $i$ to state $j$ without an arrival. Let us denote $\mathbf{D}(z)=\sum_{k=0}^{\infty} \mathbf{D}_{k} z^{k}$ and $\mathbf{D}=\sum_{k=0}^{\infty} \mathbf{D}_{k}$ is the irreducible infinitesimal generator of underlying process BMAP. Let $\overline{\boldsymbol{\pi}}$ be the stationary row vector (order $m$ ) of $\mathbf{D}$. Note that $\overline{\boldsymbol{\pi}}$ satisfies $\overline{\boldsymbol{\pi}} \mathbf{D}=\mathbf{0}$ and $\overline{\boldsymbol{\pi}} \mathbf{e}=1$, where $\mathbf{e}$ is a column vector of 1's of order $m$, respectively. The mean arrival rate, $\lambda^{*}$, and the mean batch arrival rate, $\lambda_{g}$, of the stationary BMAP is given by $\lambda^{*}=\bar{\pi}\left(\sum_{k=1}^{\infty} k \mathbf{D}_{k}\right) \mathbf{e}$, and $\lambda_{g}=\overline{\boldsymbol{\pi}}\left(\mathbf{D}-\mathbf{D}_{0}\right) \mathbf{e}=\overline{\boldsymbol{\pi}}\left(-\mathbf{D}_{0}\right) \mathbf{e}$. Also, without loss of generality, we assume that the maximum batch size of an arriving batch is $N$, i.e., we choose arrivals process as $\operatorname{BMAP}\left(\mathbf{D}_{0}, \mathbf{D}_{1}, \ldots\right)$ with $\mathbf{D}_{k}=\mathbf{0}, k \geq N+1$.

- Catastrophes arrive according to renewal process and inter-catastrophe times (ICTs) between catastrophes, are independent and identically distributed (i.i.d.) random variables (r.vs.) with distribution function $B(t)$, density function $b(t)$, Laplace-Stieltjes transform (L.-S.T.) as $B^{*}(s)$ and mean is given by $b_{1}=\mu^{-1}=-B^{*(1)}(0)$, where $\mu$ is the catastrophe arrival rate.

- When a catastrophe occurs, each individual of the population survives with a fixed probability $p \in(0,1)$, and dies with probability $q=1-p$, independent of everything. Further, the time involved in a catastrophe attack is assumed to be negligible, and there is no effect of catastrophe when the system is empty. The occurrence of catastrophes, the arrival of individuals, and the attacking process are independent of each other.

- For the stability of the system, we must have that the mean arrival rate of the BMAP to be smaller than the catastrophe rate, i.e., $\lambda^{*}<\mu p q^{-1}$, see Economou and Gómez-Corral [12]. Let us assume that $\rho=\frac{\lambda^{*} q}{\mu p}$, so for the stability of the system, we have $\rho<1$. 
Let $\pi_{n}=\left[\pi_{n, 1}, \ldots, \pi_{n, j}, \ldots, \pi_{n, m}\right]$, whose $j$ th $(1 \leq j \leq m)$ element $\pi_{n, j}$, denotes the joint probability that the population size is $n(\geq 0)$ and the phase of the arriving batch is $j$ at arbitrary epoch. And $\pi_{n}^{+}=\left[\pi_{n, 1}^{+}, \ldots, \pi_{n, j}^{+}, \ldots, \pi_{n, m}^{+}\right]$, whose $j$ th $(1 \leq j \leq m)$ element $\pi_{n, j}^{+}$, denotes the joint probability that the population size is $n(\geq 0)$ just after the occurrence of a catastrophe (post-catastrophe epoch) and the phase of the arriving batch is $j$. Further, define the VGF of $\boldsymbol{\pi}_{n}$ and $\boldsymbol{\pi}_{n}^{+}$as $\boldsymbol{\Pi}(z)=\sum_{n=0}^{\infty} \boldsymbol{\pi}_{n} z^{n}$ and $\boldsymbol{\Pi}^{+}(z)=\sum_{n=0}^{\infty} \boldsymbol{\pi}_{n}^{+} z^{n}$, respectively.

Remark 1 Throughout the paper, we assume that $f^{(k)}(\theta)$ is the $k$ th $(k \geq 1)$ derivative of $f(s)$ at $s=\theta$ and $f^{(0)}(\theta) \equiv f(\theta)$. We will use $f^{(1)}(\theta)$ and $f^{\prime}(\theta)$ interchangeably.

In the following sections, we will develop analytical tools and discuss the computational procedure for the evaluation of state probabilities at various epochs.

\section{Population Size Distribution at Various Epochs}

In this paper, we start our work with the stationary probability vector generating function (VGF) of population size distribution at post-catastrophe and arbitrary epochs, which have been derived by Economou and Gómez-Corral [12]. We obtain the population size distribution at pre-catastrophe and pre-arrival epochs which was missing in Economou and Gómez-Corral [12]. Finally, we present a detailed computing process to extract the distribution using roots method.

\section{Population Size Distribution at Post-Catastrophe and Arbitrary Epochs}

In this section, our starting point for analysis is VGF of population size distribution at postcatastrophe and arbitrary epochs which are given as (see, Eq. (8) and Eq. (40), respectively, of Economou and Gómez-Corral [12])

$$
\boldsymbol{\Pi}^{+}(z)=\boldsymbol{\pi}_{0}^{+} p(z-1)[(z-p) \mathbf{I}-q z \mathbf{A}(z)]^{-1}, \quad|z| \leq 1,
$$

and

$$
\boldsymbol{\Pi}(z)=\boldsymbol{\Pi}^{+}(z) \mathbf{S}(z), \quad|z| \leq 1,
$$

where $\mathbf{A}(z)$ is the matrix-generating function of $\mathbf{A}_{k}$ 's and is given by

$$
\mathbf{A}(z)=\sum_{k=0}^{\infty} \mathbf{A}_{k} z^{k}=\int_{0}^{\infty} \exp (\mathbf{D}(z) t) d B(t),
$$

with $\mathbf{A}_{k}$ being a square matrix of order $m$ whose $(i, j)$ th $(1 \leq i, j \leq m)$ element represents the conditional probability that given the catastrophe has occurred, and input of the underlying Markov chain of the BMAP is in phase $i$, there are $k$ individuals arrive during the inter occurrence time of two consecutive catastrophes and the input of the underlying Markov chain of the BMAP is in phase $j$ at the next catastrophe occurrence epoch.

$\mathbf{S}(z)$ is given as (for details of $\mathbf{S}(z)$, see [12, Eq. 41])

$$
\mathbf{S}(z)=\mu \int_{0}^{\infty} \exp (\mathbf{D}(z) t)(1-B(t)) d t
$$


Remark 2 In our notation, we are using $\boldsymbol{\Pi}^{+}(z), \boldsymbol{\Pi}(z)$, and $\mathbf{A}(z)$ in place of $\overline{\boldsymbol{\Pi}}^{+}(z), \overline{\boldsymbol{\Pi}}(z)$, and $\overline{\mathbf{F}}(z)$, respectively, of Economou and Gómez-Corral [12]. The notation I used in (1), denotes the identity matrix of order $m$.

\section{Population Size Distribution at Pre-Catastrophe Epoch}

Let $\pi_{n}^{c-}=\left[\pi_{n, 1}^{c-}, \ldots, \pi_{n, j}^{c-}, \ldots, \pi_{n, m}^{c-}\right]$, where $\pi_{n, j}^{c-}$ denotes the joint probability that the population size just before the occurrence of a catastrophe (pre-catastrophe epoch) is $n(\geq 0)$ and the batch arrival process is in phase $j,(1 \leq j \leq m)$. The relation between the probability vectors $\pi_{n}^{c-}$ and $\pi_{n}^{+}$is given by (for details, see Kumar and Gupta [20])

$$
\boldsymbol{\pi}_{n}^{c-}=\sum_{i=0}^{n} \boldsymbol{\pi}_{i}^{+} \mathbf{A}_{n-i}, \quad n \geq 0,
$$

which gives the VGF of the population size distribution at pre-catastrophe epoch as

$$
\boldsymbol{\Pi}^{c-}(z)=\boldsymbol{\Pi}^{+}(z) \mathbf{A}(z), \quad|z| \leq 1 .
$$

\section{Population Size Distribution at Pre-Arrival Epochs}

In this section we obtain the population size distribution at two different types of pre-arrival epochs: (i) before an arbitrary individual of an arriving batch, (ii) just before the arrival of a batch (or before the first individual of the arriving batch).

Let $\boldsymbol{\pi}_{n}^{a-}=\left[\pi_{n, 1}^{a-}, \ldots, \pi_{n, j}^{a-}, \ldots, \pi_{n, m}^{a-}\right]$ and $\pi_{n}^{b-}=\left[\pi_{n, 1}^{b-}, \ldots, \pi_{n, j}^{b-}, \ldots, \pi_{n, m}^{b-}\right]$, where $\pi_{n, j}^{a-}$ denotes the joint probability that the population size just before the arbitrary individual of an arriving batch is $n$ with the batch arrival process is in phase $j$. And $\pi_{n, j}^{b-}$ denotes the joint probability that the population size just before the arrival of a batch is $n$ with the batch arrival process is in phase $j$. The probability vectors $\pi_{n}^{a-}$ and $\pi_{n}^{b-}$ are given by (for details, see, Kumar and Gupta [18])

$$
\begin{aligned}
& \boldsymbol{\pi}_{n}^{a-}=\sum_{r=0}^{n} \boldsymbol{\pi}_{r} \widetilde{\mathbf{H}}_{n+1-r}, \quad n \geq 0, \\
& \boldsymbol{\pi}_{n}^{b-}=\boldsymbol{\pi}_{n} \widetilde{\mathbf{H}}, \quad n \geq 0,
\end{aligned}
$$

where $\widetilde{\mathbf{H}}_{k}=\frac{1}{\lambda^{*}} \sum_{i=k}^{\infty} \mathbf{D}_{i}$ and $\widetilde{\mathbf{H}}=\frac{1}{\lambda^{*}} \sum_{i=1}^{\infty} i \mathbf{D}_{i}$ (for the derivation of $\widetilde{\mathbf{H}}_{k}$ and $\widetilde{\mathbf{H}}$, see, Theorem-2 in Kumar and Gupta [18]). In the upcoming section, we present a detailed computing process to obtain the distributions at various epochs.

\section{Computing Process to Obtain the Distributions at Various Epochs}

First we discuss the computing process for getting the distribution at post-catastrophe epoch. Writing (1) as

$$
\boldsymbol{\Pi}^{+}(z)[(z-p) \mathbf{I}-q z \mathbf{A}(z)]=\boldsymbol{\pi}_{0}^{+} p(z-1) .
$$

In order to extract $\boldsymbol{\pi}_{n}^{+}$from (9), we follow inversion method of VGF, given in Chaudhry et al. [8], Gupta et al. [14], Pradhan and Gupta [23], Kumar and Gupta [18,20]. The literature shows 
that distributions having L.-S.T. as a rational function cover a wide range of distributions that arise in various applications, see Botta et al. [6]. In view of this, we consider those ICT distributions that have rational L.-S.T. of the form $h(s)=\frac{P(s)}{Q(s)}$, where degree of the polynomial $Q(s)$ is $l$ and that of the polynomial $P(s)$ is at most $l$. Moreover, for transcendental L.-S.T. of deterministic distribution, one can easily rationalized the function using Padé approximation, for details, see, Singh et al. [25,27].

Hence each element of $\mathbf{A}(z)$ is then a rational function with the same denominator, say $y(z)$. We assume that the $(i, j)$ th element of $\mathbf{A}(z)$ is $\frac{X_{i, j}(z)}{y(z)}$, i.e., $\mathbf{A}(z)=\frac{\mathbf{X}(z)}{y(z)}$, where $\mathbf{X}(z)=$ $\left[X_{i, j}(z)\right]$. Now using this expression of $\mathbf{A}(z),(9)$ can be re-written as

$$
\boldsymbol{\Pi}^{+}(z)[(z-p) y(z) \mathbf{I}-q z \mathbf{X}(z)]=\boldsymbol{\pi}_{0}^{+} y(z) p(z-1) .
$$

Let us denote the $(i, j)$ th element of $\mathbf{W}(z) \equiv\left[w_{i, j}(z)\right] \equiv[(z-p) y(z) \mathbf{I}-q z \mathbf{X}(z)]$ by $w_{i, j}(z)$ and the $j$ th element of $\mathbf{L}(z) \equiv\left[L_{1}(z) \ldots L_{j}(z) \ldots L_{m}(z)\right] \equiv \pi_{0}^{+} y(z) p$ by $L_{j}(z)$. Further, $w_{i, j}(z)$ and $L_{j}(z)$ are specified as

$$
w_{i, j}(z)= \begin{cases}(z-p) y(z)-q z X_{i, i}(z), & i=j \\ -q z X_{i, j}(z), & i \neq j,\end{cases}
$$

and

$$
L_{j}(z)=\pi_{0, j}^{+} y(z) p, \quad 1 \leq j \leq m .
$$

Now (10) can be written as

$$
\boldsymbol{\Pi}^{+}(z) \mathbf{W}(z)=(z-1) \mathbf{L}(z) .
$$

As the $j$ th $(1 \leq j \leq m)$ component of $\Pi^{+}(z)$ is $\Pi_{j}^{+}(z)$, using Cramer's rule in (11), we get the expression of $\Pi_{j}^{+}(z)$ as

$$
\Pi_{j}^{+}(z)=(z-1) \frac{\left|\mathbf{W}_{j}(z)\right|}{|\mathbf{W}(z)|}, \quad 1 \leq j \leq m,
$$

where $\mathbf{W}_{j}(z)$ is same as $\mathbf{W}(z)$ except that its $j$ th row is $\mathbf{L}(z)$. In order to fulfill the conditions of Cramer's rule and for the unique expression of $\Pi_{j}^{+}(z)$, we assume that $|\mathbf{W}(z)|$ is a non-zero polynomial in $z$. Now rewriting (12) as

$$
\Pi_{j}^{+}(z)=(z-1) \frac{\phi_{j}(z)}{\phi(z)}, \quad 1 \leq j \leq m,
$$

where $\phi_{j}(z)=\left|\mathbf{W}_{j}(z)\right|$ and $\phi(z)=|\mathbf{W}(z)|$. One may note that $\pi_{0, j}^{+}$'s are embedded in $\phi_{j}(z)$. Now our first aim is to compute the unknown vector $\pi_{0}^{+}$using the roots of $\phi(z)=0$ inside and on the unit disk $|z|=1$. As $\phi(z)=0$ has $m$ roots in $|z| \leq 1$ (see Appendix in Kumar and Gupta [20]), we assume that these $m$ roots are distinct and denote them as $\alpha_{1}, \alpha_{2}, \ldots, \alpha_{m-1}, \alpha_{m}$ with $\alpha_{m}=1$ (for repeated roots see Remark 3).

\section{Determination of the Unknown Vector $\pi_{0}^{+}$}

All the roots that lies inside the unit circle, i.e., $\alpha_{1}, \alpha_{2}, \ldots, \alpha_{m-1}$ and $\alpha_{m}$ must be the zeros of the numerator of (13) since each component $\Pi_{j}^{+}(z)$ of the VGF $\boldsymbol{\Pi}^{+}(z)$ given in (13) is analytic in $|z| \leq 1$ and hence one can determine the unknown vector $\pi_{0}^{+}$by considering any 
one component of $\boldsymbol{\Pi}^{+}(z)$, say $\Pi_{l}^{+}(z)(1 \leq l \leq m)$. So we get $m$ simultaneous equations in $m$ unknowns such that

$$
\begin{aligned}
\phi_{l}\left(\alpha_{i}\right) & =0, \quad 1 \leq i \leq m-1, \\
\phi_{l}(1) & =\bar{\pi}_{l} \phi^{\prime}(1) .
\end{aligned}
$$

After solving the system of Eqs. (14) and (15), we get the unknown vector $\pi_{0}^{+}$. Now after substituting the known vector $\pi_{0}^{+}$in (13), we get $\Pi_{j}^{+}(z)$ in the form of completely known polynomials both in the numerator and the denominator. Rewriting (13) as

$$
\Pi_{j}^{+}(z)=(z-1) Y_{j}(z), \quad 1 \leq j \leq m,
$$

where

$$
Y_{j}(z)=\frac{\phi_{j}(z)}{\phi(z)}, \quad 1 \leq j \leq m .
$$

As the degree of the numerator of (17) is less than or equal to the degree of the denominator, let $\phi(z)=0$ has $d$ distinct roots outside $|z|=1$, viz. $v_{i}, 1 \leq i \leq d$ (for repeated roots see Remark 3). Now applying the partial-fraction method on $Y_{j}(z)$, we obtain

$$
Y_{j}(z)=\sum_{k=0}^{\infty} y_{k, j} z^{k}=c_{0, j}+\sum_{i=1}^{d} \frac{c_{i, j}}{\left(z-v_{i}\right)}, \quad 1 \leq j \leq m .
$$

Now collecting the coefficient of $z^{k}, k \geq 0$ from both sides of (18), we get

$$
y_{k, j}= \begin{cases}c_{0, j}-\sum_{i=1}^{d} \frac{c_{i, j}}{v_{i}}, & k=0 \\ -\sum_{i=1}^{d} \frac{c_{i, j}}{v_{i}^{k+1}}, & k \geq 1,\end{cases}
$$

where

$$
c_{i, j}= \begin{cases}\operatorname{quo}\left(\phi_{j}(z), \phi(z)\right), & i=0 \\ \frac{\phi_{j}\left(v_{i}\right)}{\phi^{\prime}\left(v_{i}\right)}, & i=1, \ldots, d\end{cases}
$$

Now using (19) in (16) and then collecting the coefficient of $z^{k}$ from both sides of (16), we finally obtain for $1 \leq j \leq m$

$$
\begin{aligned}
& \pi_{0, j}^{+}=-y_{0, j} \\
& \pi_{k, j}^{+}=y_{k-1, j}-y_{k, j}, \quad k \geq 1 .
\end{aligned}
$$

This completes the computing process of the population size distribution at post-catastrophe epoch.

Remark 3 If there are repeated roots, a slight modification is needed in the computing process, see, e.g., Gupta et al. [14], Pradhan and Gupta [23].

In order to obtain population size distribution at arbitrary epoch, from (2) we have

$$
\boldsymbol{\pi}_{k}=\sum_{l=0}^{k} \boldsymbol{\pi}_{l}^{+} \mathbf{S}_{l-k}, \quad k \geq 0 .
$$


The population size distribution at pre-catastrophe and pre-arrival epochs can be obtained using (5), (7), and (8), respectively.

After obtaining all the distribution at various epochs, we are in position to evaluate the factorial moments of the population size at each epoch which are given in the upcoming section.

\section{Factorial Moments}

In this section we obtain factorial moments of the population size at various epochs.

\section{Factorial Moments of the Population Size Using the Probability Distributions}

Let $m_{n}^{+}, m_{n}, m_{n}^{c-}, m_{n}^{a-}, m_{n}^{b-}$ denotes the $n$th factorial moments of the population size at postcatastrophe, arbitrary, pre-catastrophe and pre-arrival epochs, respectively. The $n$th $(\geq 1)$ factorial moment at post-catastrophe epoch is given by

$$
m_{n}^{+}=\left(\sum_{i=n}^{\infty} i(i-1) \ldots(i-n+1) \pi_{i}^{+}\right) \boldsymbol{e}, \quad n \geq 1 .
$$

Now to obtain $m_{n}, m_{n}^{c-}, m_{n}^{a-}, m_{n}^{b-}$, simply replace $\boldsymbol{\pi}_{i}^{+}$by $\boldsymbol{\pi}_{i}, \boldsymbol{\pi}_{i}^{c-}, \boldsymbol{\pi}_{i}^{a-}, \boldsymbol{\pi}_{i}^{b-}$, respectively. For $n=1$, we get the mean population size at various epochs. In the following section we obtain factorial moments of the population size using VGF. It also performs a check over the computing process.

\section{Factorial Moments of the Population Size Using VGF}

Theorem 1 Factorial moment of the population size at post-catastrophe epoch $\left(m_{n}^{+}, n \geq 1\right)$ is given by

$$
\begin{aligned}
& m_{1}^{+}=\left(\frac{p}{q}-\frac{\lambda^{*}}{\mu}\right)^{-1}\left(\frac{1}{2} \bar{\pi} \mathbf{f}_{2}+\left(\frac{p}{q} \pi_{0}^{+}+\bar{\pi} \mathbf{A}^{(1)}(1)\right) \widetilde{\mathbf{Z}} \mathbf{f}_{1}+\frac{\lambda^{*}}{\mu}\left(1-\frac{p}{q}\right)\right) \\
& m_{n}^{+}=\left(\frac{p}{q}-\frac{\lambda^{*}}{\mu}\right)^{-1}\left(\omega_{n} \mathbf{f}_{1}+\frac{1}{n+1} \sum_{k=2}^{n+1}\left(\begin{array}{c}
n+1 \\
k
\end{array}\right) \Pi^{+(n+1-k)}(1)\left(\mathbf{f}_{k}+k \mathbf{f}_{k-1}\right)\right), \quad n \geq 2
\end{aligned}
$$

where

$$
\begin{aligned}
\mathbf{f}_{n} & =\mathbf{A}^{(n)}(1) \boldsymbol{e}, \quad n \geq 1, \\
\widetilde{\mathbf{Z}} & =(\mathbf{I}-\mathbf{A}(1)+\boldsymbol{e} \overline{\boldsymbol{\pi}})^{-1}, \\
\boldsymbol{\omega}_{n} & =\frac{-n}{1-p} \boldsymbol{\Pi}^{+(n-1)}(1)\left(\mathbf{I}-q\left(\mathbf{A}(1)+\mathbf{A}^{(1)}(1)\right)\right) \widetilde{\mathbf{Z}} \\
& \quad+\sum_{k=2}^{n}\left(\begin{array}{l}
n \\
k
\end{array}\right) \boldsymbol{\Pi}^{+(n-k)}(1)\left(\mathbf{A}^{(k)}(1)+k \mathbf{A}^{(k-1)}(1)\right) \widetilde{\mathbf{Z}}, \quad n \geq 2,
\end{aligned}
$$




$$
\begin{aligned}
& \boldsymbol{\Pi}^{+(1)}(1)=\left(m_{1}^{+}-\frac{p}{q}\right) \overline{\boldsymbol{\pi}}+\left(\frac{p}{q} \boldsymbol{\pi}_{0}^{+}+\overline{\boldsymbol{\pi}} \mathbf{A}^{(1)}(1)\right) \widetilde{\mathbf{Z}}, \\
& \boldsymbol{\Pi}^{+(n)}(1)=m_{n}^{+} \overline{\boldsymbol{\pi}}+\boldsymbol{\omega}_{n}, \quad n \geq 2 .
\end{aligned}
$$

Proof The proof of the theorem can be found in [12, Theorem-3.1.2.]. However, for the sake of completeness and easy understanding for the readers, a detailed proof of the theorem is given below.

Equation (1) can be written as

$$
\boldsymbol{\Pi}^{+}(z)((z-p) \mathbf{I}-q z \mathbf{A}(z))=\boldsymbol{\pi}_{0}^{+} p(z-1), \quad|z| \leq 1 .
$$

Taking derivative of (26) $n$ times w.r.t. $z$, we get following equations for $n=1$, and $n \geq 2$, respectively

$$
\begin{aligned}
& \boldsymbol{\Pi}^{+(1)}(z)((z-p) \mathbf{I}-q z \mathbf{A}(z))+\boldsymbol{\Pi}^{+}(z)\left(\mathbf{I}-q\left(z \mathbf{A}^{(1)}(z)+\mathbf{A}(z)\right)\right)=\boldsymbol{\pi}_{0}^{+} p . \\
& \boldsymbol{\Pi}^{+(n)}(z)((z-p) \mathbf{I}-q z \mathbf{A}(z))+n \boldsymbol{\Pi}^{+(n-1)}(z)\left(\mathbf{I}-q\left(z \mathbf{A}^{(1)}(z)+\mathbf{A}(z)\right)\right) \\
& \quad=q \sum_{k=2}^{n}\left(\begin{array}{l}
n \\
k
\end{array}\right) \boldsymbol{\Pi}^{+(n-k)}(z)\left(z \mathbf{A}^{(k)}(z)+k \mathbf{A}^{(k-1)}(z)\right), \quad n \geq 2 .
\end{aligned}
$$

Putting $z=1$ in (26), we get

$$
\boldsymbol{\Pi}^{+}(1)(\mathbf{I}-\mathbf{A}(1))=\mathbf{0} .
$$

Now using the fact that $\boldsymbol{\Pi}^{+}(1)=\overline{\boldsymbol{\pi}}$, (29) gives

$$
\overline{\boldsymbol{\pi}}(\mathbf{I}-\mathbf{A}(1)+\mathbf{e} \overline{\boldsymbol{\pi}})=\overline{\boldsymbol{\pi}} .
$$

Now setting $z=1$ in (27) and using (29) with $\boldsymbol{\Pi}^{+}(1)=\overline{\boldsymbol{\pi}}$, we obtain

$$
\boldsymbol{\Pi}^{+(1)}(1)(\mathbf{I}-\mathbf{A}(1))=\frac{p}{q}\left(\boldsymbol{\pi}_{0}^{+}-\overline{\boldsymbol{\pi}}\right)+\overline{\boldsymbol{\pi}}\left(\mathbf{A}^{(1)}(1)\right) .
$$

Now by the definition of $m_{n}^{+}$, we have

$$
\boldsymbol{\Pi}^{+(n)}(1) \mathbf{e} \overline{\boldsymbol{\pi}}=m_{n}^{+} \overline{\boldsymbol{\pi}}, \quad n \geq 1 .
$$

Adding (31) and (32) for $n=1$, we get

$$
\boldsymbol{\Pi}^{+(1)}(1)(\mathbf{I}-\mathbf{A}(1)+\mathbf{e} \overline{\boldsymbol{\pi}})=m_{1}^{+} \overline{\boldsymbol{\pi}}+\frac{p}{q}\left(\boldsymbol{\pi}_{0}^{+}-\overline{\boldsymbol{\pi}}\right)+\overline{\boldsymbol{\pi}}\left(\mathbf{A}^{(1)}(1)\right) .
$$

After simplification and using (30), (33) gives

$$
\boldsymbol{\Pi}^{+(1)}(1)=\left(m_{1}^{+}-\frac{p}{q}\right) \overline{\boldsymbol{\pi}}+\left(\frac{p}{q} \boldsymbol{\pi}_{0}^{+}+\overline{\boldsymbol{\pi}} \mathbf{A}^{(1)}(1)\right) \widetilde{\mathbf{Z}},
$$

where $\widetilde{\mathbf{Z}}=(\mathbf{I}-\mathbf{A}(1)+\mathbf{e} \overline{\boldsymbol{\pi}})^{-1}$. Now setting $z=1$ in (28) for $n=2$ and then postmultiplying it by $\mathbf{e}$, we obtain

$$
m_{1}^{+}=\frac{q}{2 p}\left(2 \boldsymbol{\Pi}^{+(1)}(1) \mathbf{f}_{1}+\overline{\boldsymbol{\pi}}\left(\mathbf{f}_{2}+2 \mathbf{f}_{1}\right)\right) .
$$

Now using (34) in (35), we get

$$
m_{1}^{+}=\frac{q}{2 p}\left(2\left(\left(m_{1}^{+}-\frac{p}{q}\right) \overline{\boldsymbol{\pi}}+\left(\frac{p}{q} \boldsymbol{\pi}_{0}^{+}+\overline{\boldsymbol{\pi}} \mathbf{A}^{(1)}(1)\right) \widetilde{\mathbf{Z}}\right) \mathbf{f}_{1}+\overline{\boldsymbol{\pi}}\left(\mathbf{f}_{2}+2 \mathbf{f}_{1}\right)\right) .
$$


After simplification (36) gives

$$
m_{1}^{+}=\left(\frac{p}{q}-\frac{\lambda^{*}}{\mu}\right)^{-1}\left(\frac{1}{2} \bar{\pi} \mathbf{f}_{2}+\left(\frac{p}{q} \pi_{0}^{+}+\bar{\pi} \mathbf{A}^{(1)}(1)\right) \widetilde{\mathbf{Z}} \mathbf{f}_{1}+\frac{\lambda^{*}}{\mu}\left(1-\frac{p}{q}\right)\right) .
$$

Hence (24) is proved.

Again setting $z=1$ in (28), we get

$$
\begin{gathered}
\boldsymbol{\Pi}^{+(n)}(1) q(\mathbf{I}-\mathbf{A}(1))+n \boldsymbol{\Pi}^{+(n-1)}(1)\left(\mathbf{I}-q\left(\mathbf{A}^{(1)}(1)+\mathbf{A}(1)\right)\right) \\
=q \sum_{k=2}^{n}\left(\begin{array}{l}
n \\
k
\end{array}\right) \boldsymbol{\Pi}^{+(n-k)}(1)\left(\mathbf{A}^{(k)}(1)+k \mathbf{A}^{(k-1)}(1)\right), \quad n \geq 2 .
\end{gathered}
$$

That gives

$$
\begin{aligned}
\boldsymbol{\Pi}^{+(n)}(1)(\mathbf{I}-\mathbf{A}(1))= & \frac{-n}{q} \boldsymbol{\Pi}^{+(n-1)}(1)\left(\mathbf{I}-q\left(\mathbf{A}^{(1)}(1)+\mathbf{A}(1)\right)\right) \\
& +\sum_{k=2}^{n}\left(\begin{array}{l}
n \\
k
\end{array}\right) \boldsymbol{\Pi}^{+(n-k)}(1)\left(\mathbf{A}^{(k)}(1)+k \mathbf{A}^{(k-1)}(1)\right), \quad n \geq 2 .
\end{aligned}
$$

Adding (39) and (32), we obtain

$$
\begin{aligned}
\boldsymbol{\Pi}^{+(n)}(1)(\mathbf{I}-\mathbf{A}(1)+\mathbf{e} \overline{\boldsymbol{\pi}})= & m_{n}^{+} \overline{\boldsymbol{\pi}}+\frac{-n}{q} \boldsymbol{\Pi}^{+(n-1)}(1)\left(\mathbf{I}-q\left(\mathbf{A}^{(1)}(1)+\mathbf{A}(1)\right)\right) \\
& +\sum_{k=2}^{n}\left(\begin{array}{l}
n \\
k
\end{array}\right) \boldsymbol{\Pi}^{+(n-k)}(1)\left(\mathbf{A}^{(k)}(1)+k \mathbf{A}^{(k-1)}(1)\right), \quad n \geq 2 .
\end{aligned}
$$

That implies

$$
\boldsymbol{\Pi}^{+(n)}(1)=m_{n}^{+\bar{\pi}}+\boldsymbol{\omega}_{n}, \quad n \geq 2,
$$

where

$$
\begin{aligned}
\boldsymbol{\omega}_{n}= & \frac{-n}{q} \boldsymbol{\Pi}^{+(n-1)}(1)\left(\mathbf{I}-q\left(\mathbf{A}^{(1)}(1)+\mathbf{A}(1)\right)\right) \widetilde{\mathbf{Z}} \\
& +\sum_{k=2}^{n}\left(\begin{array}{l}
n \\
k
\end{array}\right) \boldsymbol{\Pi}^{+(n-k)}(1)\left(\mathbf{A}^{(k)}(1)+k \mathbf{A}^{(k-1)}(1)\right) \widetilde{\mathbf{Z}}, \quad n \geq 2 .
\end{aligned}
$$

Post-multiplying (38) by e, we obtain

$$
p m_{n-1}^{+}=\boldsymbol{\Pi}^{+(n-1)}(1) q \mathbf{A}^{(1)}(1) \mathbf{e}+\frac{q}{n} \sum_{k=2}^{n}\left(\begin{array}{l}
n \\
k
\end{array}\right) \boldsymbol{\Pi}^{+(n-k)}(1)\left(\mathbf{A}^{(k)}(1)+k \mathbf{A}^{(k-1)}(1)\right) \mathbf{e}, n \geq 3 .
$$

Using (41) in (42), we get

$$
p m_{n-1}^{+}=\left(m_{n-1}^{+} \bar{\pi}+\omega_{n-1}\right) q \mathbf{A}^{(1)}(1) \mathbf{e}+\frac{q}{n} \sum_{k=2}^{n}\left(\begin{array}{l}
n \\
k
\end{array}\right) \Pi^{+(n-k)}(1)\left(\mathbf{A}^{(k)}(1)+k \mathbf{A}^{(k-1)}(1)\right) \mathbf{e}, n \geq 3 .
$$


(43), gives

$$
\begin{gathered}
m_{n-1}^{+}=\left(m_{n-1}^{+} \bar{\pi}+\omega_{n-1}\right) q \mathbf{A}^{(1)}(1) \mathbf{e}+\frac{q}{n} \sum_{k=2}^{n}\left(\begin{array}{l}
n \\
k
\end{array}\right) \Pi^{+(n-k)}(1)\left(\mathbf{A}^{(k)}(1)+k \mathbf{A}^{(k-1)}(1)\right) \mathbf{e}, n \geq 3 \\
m_{n-1}^{+}=\left(\frac{p}{q}-\frac{\lambda^{*}}{\mu}\right)^{-1}\left(\omega_{n-1} \mathbf{f}_{1}+\frac{1}{n} \sum_{k=2}^{n}\left(\begin{array}{l}
n \\
k
\end{array}\right) \Pi^{+(n-k)}(1)\left(\mathbf{f}_{k}+k \mathbf{f}_{k-1}\right)\right), n \geq 3 .(44)
\end{gathered}
$$

Equation (44) can also be written as

$$
m_{n}^{+}=\left(\frac{p}{q}-\frac{\lambda^{*}}{\mu}\right)^{-1}\left(\omega_{n} \mathbf{f}_{1}+\frac{1}{n+1} \sum_{k=2}^{n+1}\left(\begin{array}{c}
n+1 \\
k
\end{array}\right) \Pi^{+(n+1-k)}(1)\left(\mathbf{f}_{k}+k \mathbf{f}_{k-1}\right)\right), \quad n \geq 2
$$

which proves the theorem.

Theorem 2 Factorial moment of the population size at arbitrary $\left(m_{n}\right)$, and pre-catastrophe $\left(m_{n}^{c-}\right)$ epochs are given as

$$
\begin{gathered}
m_{n}=m_{n}^{+}+\sum_{i=1}^{n-1}\left(\begin{array}{l}
n \\
i
\end{array}\right) \boldsymbol{\Pi}^{+(n-i)}(1) \mathbf{k}_{i}+\bar{\pi} \mathbf{k}_{n}, \quad n \geq 1 . \\
m_{n}^{c-}=m_{n}^{+}+\sum_{i=1}^{n-1}\left(\begin{array}{l}
n \\
i
\end{array}\right) \boldsymbol{\Pi}^{+(n-i)}(1) \mathbf{f}_{i}+\bar{\pi} \mathbf{f}_{n}, \quad n \geq 1 .
\end{gathered}
$$

where

$$
\mathbf{k}_{i}=\mathbf{S}^{(i)}(1) \boldsymbol{e}, \quad i \geq 1 .
$$

Proof Differentiating (2) and (6) $n$ times w.r.t. $z$, we get

$$
\begin{gathered}
\boldsymbol{\Pi}^{(n)}(z)=\boldsymbol{\Pi}^{+(n)}(z) \mathbf{S}(z)+\sum_{i=1}^{n-1}\left(\begin{array}{l}
n \\
i
\end{array}\right) \boldsymbol{\Pi}^{+(n-i)}(z) \mathbf{S}^{(i)}(z)+\boldsymbol{\Pi}^{+}(z) \mathbf{S}^{(n)}(z) . \\
\boldsymbol{\Pi}^{c-(n)}(z)=\boldsymbol{\Pi}^{+(n)}(z) \mathbf{A}(z)+\sum_{i=1}^{n-1}\left(\begin{array}{l}
n \\
i
\end{array}\right) \boldsymbol{\Pi}^{+(n-i)}(z) \mathbf{A}^{(i)}(z)+\boldsymbol{\Pi}^{+}(z) \mathbf{A}^{(n)}(z) .
\end{gathered}
$$

Now setting $z=1$ and post multiplying by $\boldsymbol{e}$ in (47)-(48), we obtain

$$
\begin{gathered}
m_{n}=\boldsymbol{\Pi}^{(n)}(1) \boldsymbol{e}=\boldsymbol{\Pi}^{+(n)}(1) \boldsymbol{e}+\sum_{i=1}^{n-1}\left(\begin{array}{l}
n \\
i
\end{array}\right) \boldsymbol{\Pi}^{+(n-i)}(1) \mathbf{S}^{(i)}(1) \boldsymbol{e}+\overline{\boldsymbol{\pi}} \mathbf{S}^{(n)}(1) \boldsymbol{e} \\
m_{n}^{c-}=\boldsymbol{\Pi}^{c-(n)}(1) \boldsymbol{e}=\boldsymbol{\Pi}^{+(n)}(1) \boldsymbol{e}+\sum_{i=1}^{n-1}\left(\begin{array}{l}
n \\
i
\end{array}\right) \boldsymbol{\Pi}^{+(n-i)}(1) \mathbf{A}^{(i)}(1) \boldsymbol{e}+\overline{\boldsymbol{\pi}} \mathbf{A}^{(n)}(1) \boldsymbol{e}
\end{gathered}
$$

Now using $\mathbf{k}_{i}=\mathbf{S}^{(i)}(1) \boldsymbol{e}$ and $\mathbf{f}_{i}=\mathbf{A}^{(i)}(1) \boldsymbol{e}$ for $i \geq 1$, (49) and (50) implies

$$
\begin{gathered}
m_{n}=m_{n}^{+}+\sum_{i=1}^{n-1}\left(\begin{array}{l}
n \\
i
\end{array}\right) \Pi^{+(n-i)}(1) \mathbf{k}_{i}+\bar{\pi} \mathbf{k}_{n}, \quad n \geq 1 . \\
m_{n}^{c-}=m_{n}^{+}+\sum_{i=1}^{n-1}\left(\begin{array}{l}
n \\
i
\end{array}\right) \Pi^{+(n-i)}(1) \mathbf{f}_{i}+\bar{\pi} \mathbf{f}_{n}, \quad n \geq 1
\end{gathered}
$$


Hence the theorem is proved.

Remark 4 As the population size distribution at both the pre-arrival epochs are given in terms of probability vectors, so $m_{n}^{a-}$ and $m_{n}^{b-}$ can be obtained using (23).

In the following sections, we will discuss some special cases and a few numerical aspects of the model.

\section{Special Cases}

In this section, we discuss some special cases of the model and match our results with the existing one.

\section{Poisson Arrival Process Subject to Poisson Generated Geometric Catastrophes}

Here, we assume that individuals as well as catastrophes occur as per the Poisson process. For this we have $m=1, \mathbf{D}_{0}=[-\lambda], \mathbf{D}_{1}=[\lambda], \mathbf{D}_{i}=[0], i \geq 2$ and $\mathbf{A}(z)(\equiv A(z))=$ $\frac{\mu}{\mu+\lambda-\lambda z}, \mathbf{S}(z)(\equiv S(z))=A(z)$. Thus (1), (2), (7) and (8) becomes

$$
\begin{aligned}
\Pi^{+}(z)\left(\equiv \Pi^{+}(z)\right) & =\frac{\pi_{0}^{+} p(z-1)}{(z-p)-q z A(z)}=\frac{\pi_{0}^{+} p(\mu+\lambda-\lambda z)}{p(\mu+\lambda)-\lambda z}, \\
\Pi(z)(\equiv \Pi(z)) & =\Pi^{+}(z) S(z)=\frac{\pi_{0}^{+} p \mu}{p(\mu+\lambda)-\lambda z}, \\
\pi_{n}^{-} & =\pi_{n}^{a-}=\pi_{n}^{b-}=\pi_{n}, n \geq 0 .
\end{aligned}
$$

Here $\pi_{n}^{-}$denotes the probability that the population size in $n$ just before the arrival of individual. Since we are assuming that individuals arrive singly, so $\pi_{n}^{-}=\pi_{n}^{a-}=\pi_{n}^{b-}$. After simplification (53) gives

$$
\begin{aligned}
& \pi_{0}^{+}=1-\frac{\lambda q}{\mu p} \\
& \pi_{n}^{+}=q\left(1-\frac{\lambda q}{\mu p}\right)\left(\frac{\lambda}{p(\lambda+\mu)}\right)^{n}, n \geq 1,
\end{aligned}
$$

and using (56) in (54), we get

$$
\pi_{n}=\left(1-\frac{\lambda}{p(\mu+\lambda)}\right)\left(\frac{\lambda}{p(\lambda+\mu)}\right)^{n}, n \geq 0,
$$

The results given in (55) and (58) match exactly with the one given in [5, Eq. 30]. It can be observed that in order to obtain the results at arbitrary and pre-arrival epochs, we have used the post-catastrophe epoch probabilities.

\section{Erlang $\left(E_{4}\right)$ Batch Arrival Process Subject to Poisson Generated Geometric Catastrophes}

Here, we assume that individuals arrive in batches according to the Erlang $\left(E_{4}\right)$ process and catastrophes occur as per the Poisson process. For this the matrix representation of $E_{4}$ is chosen as $m=4, \lambda=20$, 
$\mathbf{D}_{0}=\left[\begin{array}{cccc}-\lambda & \lambda & 0 & 0 \\ 0 & -\lambda & \lambda & 0 \\ 0 & 0 & -\lambda & \lambda \\ 0 & 0 & 0 & -\lambda\end{array}\right], \mathbf{D}_{1}=0.2\left[\begin{array}{llll}0 & 0 & 0 & 0 \\ 0 & 0 & 0 & 0 \\ 0 & 0 & 0 & 0 \\ \lambda & 0 & 0 & 0\end{array}\right], \mathbf{D}_{3}=0.4\left[\begin{array}{llll}0 & 0 & 0 & 0 \\ 0 & 0 & 0 & 0 \\ 0 & 0 & 0 & 0 \\ \lambda & 0 & 0 & 0\end{array}\right]$,

$\mathbf{D}_{6}=0.3\left[\begin{array}{llll}0 & 0 & 0 & 0 \\ 0 & 0 & 0 & 0 \\ 0 & 0 & 0 & 0 \\ \lambda & 0 & 0 & 0\end{array}\right], \mathbf{D}_{10}=0.1\left[\begin{array}{llll}0 & 0 & 0 & 0 \\ 0 & 0 & 0 & 0 \\ 0 & 0 & 0 & 0 \\ \lambda & 0 & 0 & 0\end{array}\right]$, and $\mathbf{D}_{i},(i \neq 0,1,3,6,10)$ are zero matri-

ces of order 4 . Further, we have chosen $\mu=15$ and $p=0.64$. The population size distribution at various epochs is given in Table 1 . The results given in Table 1 for arbitrary and just before the arrival of a batch match exactly with the one given in [5, Table-1] for $G I=E_{4}$, i.e., $\boldsymbol{\pi}_{n} \boldsymbol{e}=P_{n}$ and $\boldsymbol{\pi}_{n}^{b-} \boldsymbol{e}=P_{n}^{-}$for $n \geq 0$.

\section{Hyper-Exponential $\left(\mathrm{HE}_{2}\right)$ Batch Arrival Process Subject to Poisson Generated Geometric Catastrophes}

Here, we assume that individuals arrive in batches according to the Hyper-exponential $\left(H E_{2}\right)$ and catastrophes occur as per the Poisson process. For this the matrix representation of $\mathrm{HE}_{2}$ is chosen as $m=2$,

$\mathbf{D}_{0}=\left[\begin{array}{cc}-\frac{20}{7} & 0 \\ 0 & -20\end{array}\right], \mathbf{D}_{1}=0.8\left[\begin{array}{cc}\frac{20}{21} & \frac{40}{21} \\ \frac{20}{3} & \frac{40}{3}\end{array}\right], \mathbf{D}_{3}=0.15\left[\begin{array}{cc}\frac{20}{21} & \frac{40}{21} \\ \frac{20}{3} & \frac{40}{3}\end{array}\right]$,
$\mathbf{D}_{8}=0.05\left[\begin{array}{cc}\frac{20}{21} & \frac{40}{21} \\ \frac{20}{3} & \frac{40}{3}\end{array}\right]$, and $\mathbf{D}_{i},(i \neq 0,1,3,8)$ are zero matrices of order 2. Further, we have chosen $\mu=35$ and $p=0.4$. The population size distribution at various epochs is given in Table 2. The results given in Table 2 for arbitrary and just before the arrival of a batch match exactly with the one given in [5, Table-2] for $G I=H E_{2}$, i.e., $\boldsymbol{\pi}_{n} \boldsymbol{e}=P_{n}$ and $\boldsymbol{\pi}_{n}^{b-} \boldsymbol{e}=P_{n}^{-}$for $n \geq 0$.

\section{Numerical Results}

In this section we present numerical work based on computational procedure discussed in Sect. 4 by considering arrival process $\operatorname{BMAP}\left(\mathbf{D}_{0}, \mathbf{D}_{1}, \ldots, \mathbf{D}_{N}\right)$ and various ICT distributions. All the calculations have been performed using MAPLE software on a PC having configurations as Intel(R) Core i5-6500U processor @ $2.30 \mathrm{GHz}$ with 8GB RAM in Windows 10 environment. For numerical examples we have chosen the following set of matrices $\mathbf{D}_{i}, 0 \leq i \leq N$, of order $m=4$ with $N=5$. These matrices are considered for examples 1-3. 


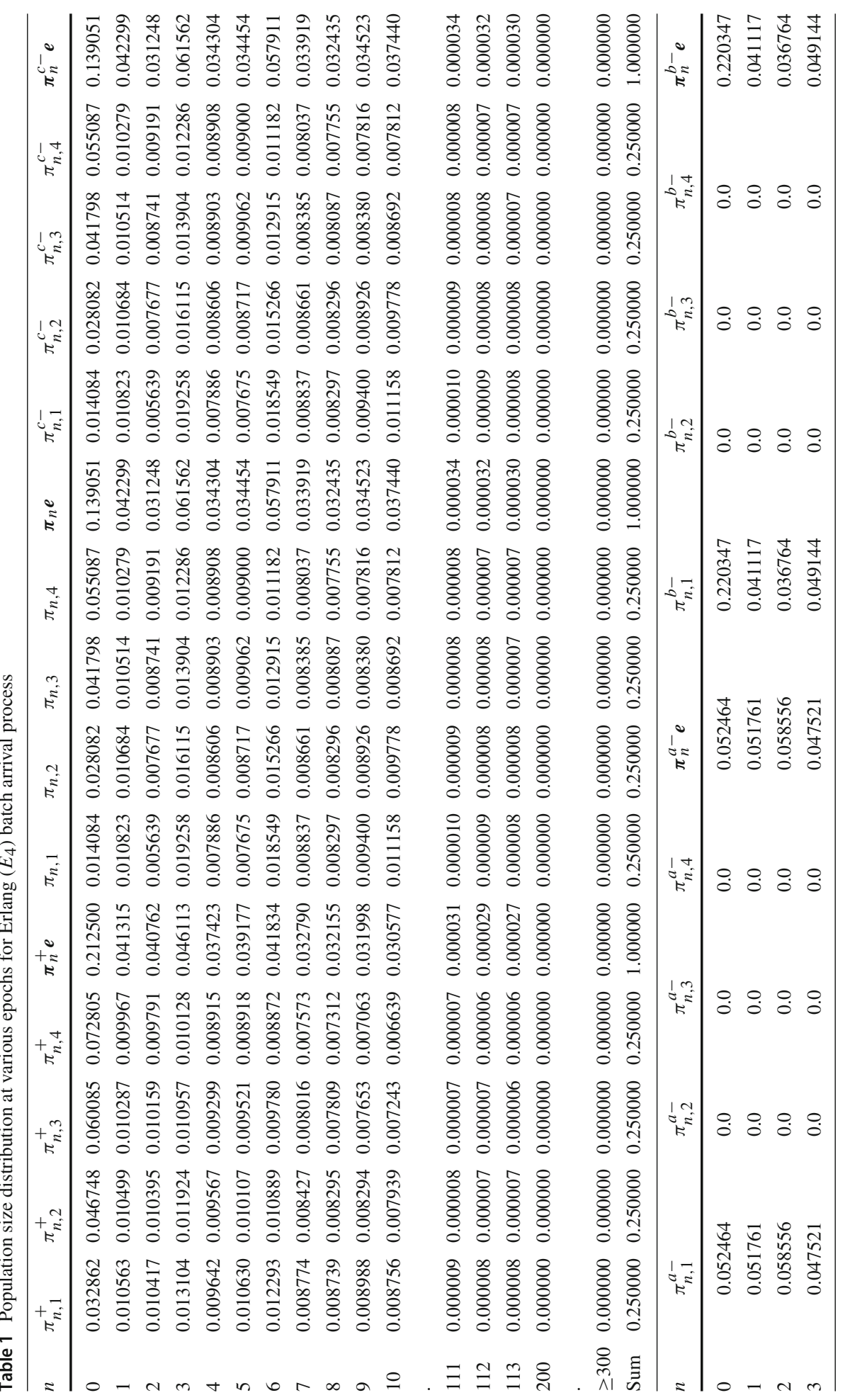




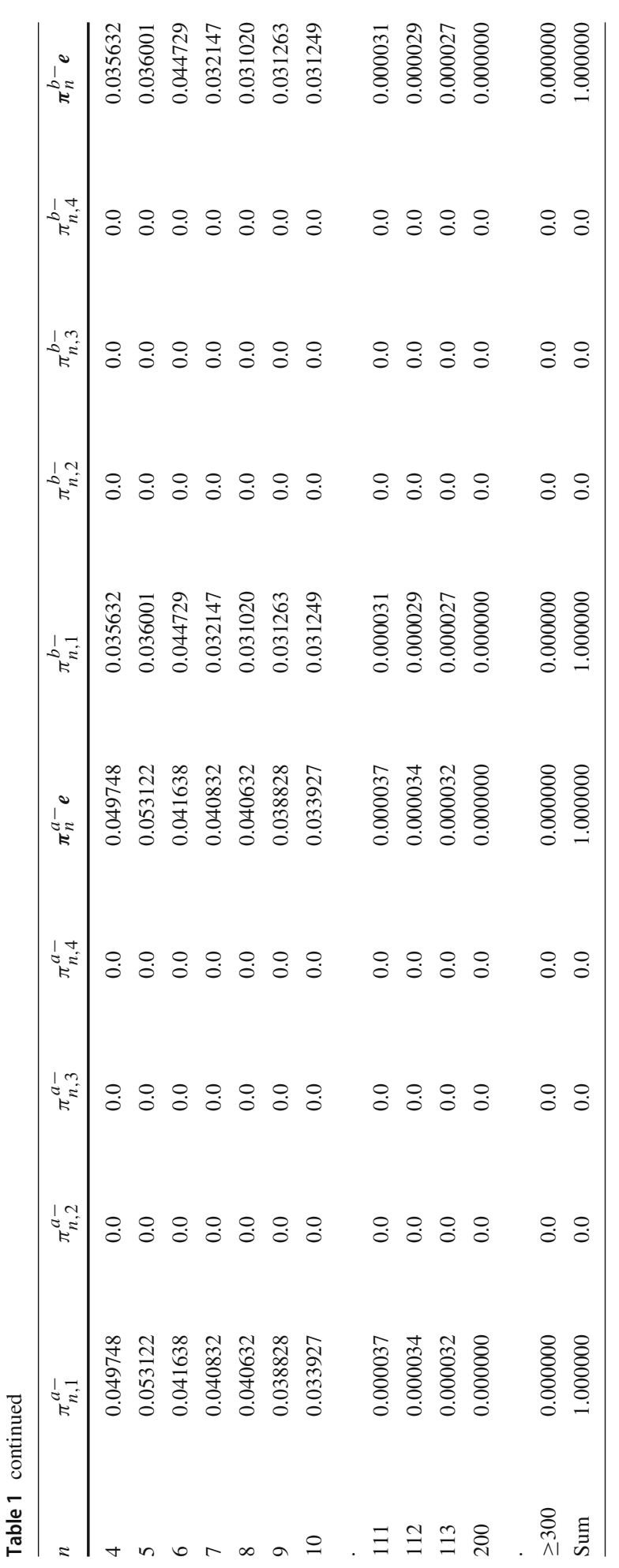




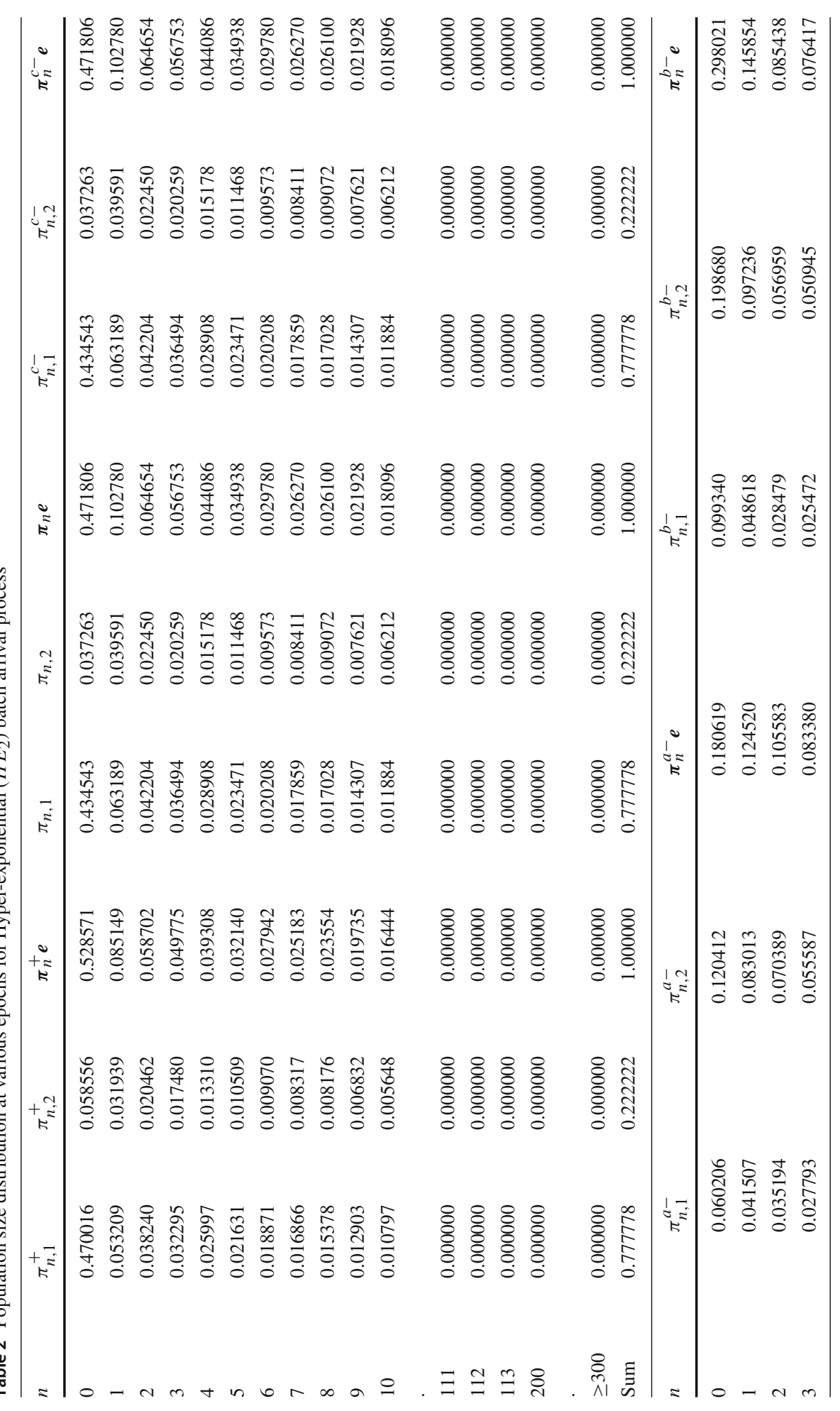




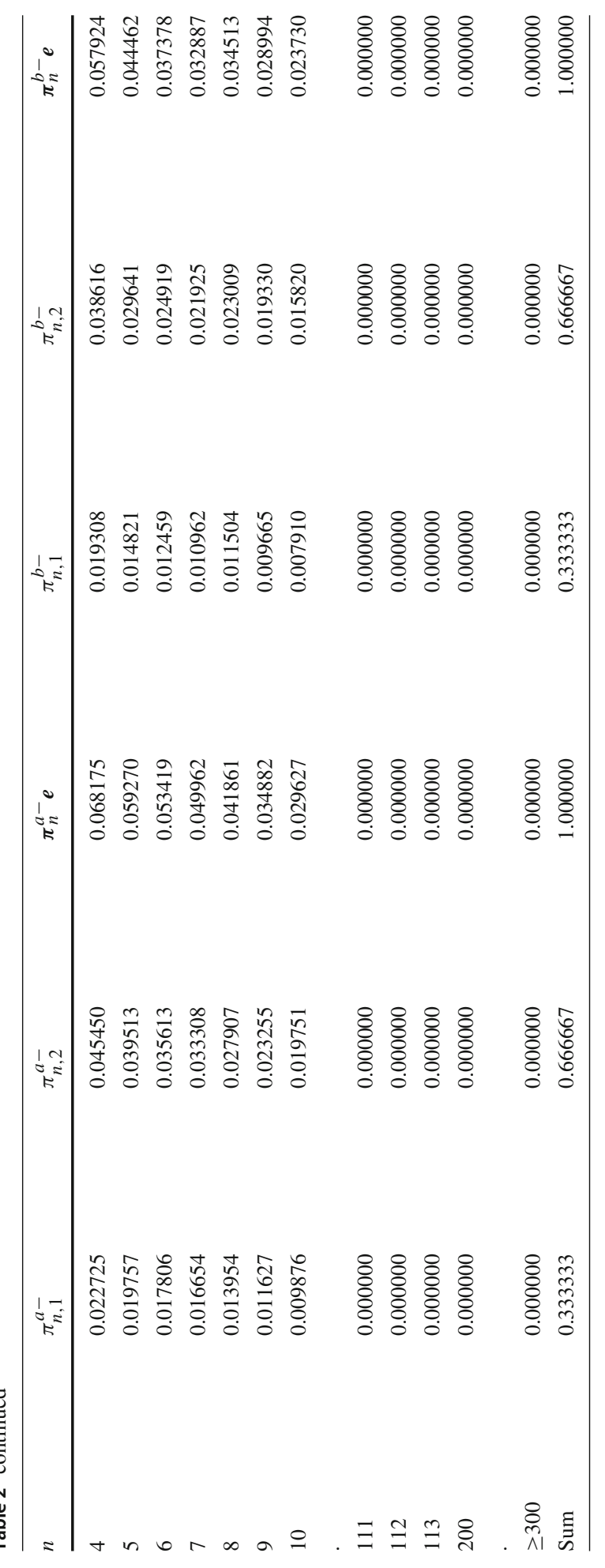




$$
\begin{aligned}
& \mathbf{D}_{0}= {\left[\begin{array}{cccc}
-0.204360 & 0.172920 & 0.015720 & 0.0 \\
0.015720 & -0.235800 & 0.0 & 0.015720 \\
0.172920 & 0.0 & -0.220080 & 0.015720 \\
0.015720 & 0.015720 & 0.015720 & -1.493400
\end{array}\right], } \\
& \mathbf{D}_{1}= {\left[\begin{array}{cccc}
0.0 & 0.0 & 0.00333264 & 0.0 \\
0.03665904 & 0.00333264 & 0.00333264 & 0.0 \\
0.00333264 & 0.0 & 0.00333264 & 0.0 \\
0.0 & 0.0 & 0.00333264 & 0.30327024
\end{array}\right], } \\
& \mathbf{D}_{2}= {\left[\begin{array}{cccc}
0.0 & 0.0 & 0.00342696 & 0.0 \\
0.03769656 & 0.00342696 & 0.00342696 & 0.0 \\
0.00342696 & 0.0 & 0.00342696 & 0.0 \\
0.0 & 0.0 & 0.00342696 & 0.31185336
\end{array}\right], } \\
& \mathbf{D}_{3}=\left[\begin{array}{cccc}
0.0 & 0.0 & 0.00268812 & 0.0 \\
0.02956932 & 0.00268812 & 0.00268812 & 0.0 \\
0.00268812 & 0.0 & 0.00268812 & 0.0 \\
0.0 & 0.0 & 0.00268812 & 0.24461892
\end{array}\right], \\
& \mathbf{D}_{4}=\left[\begin{array}{cccc}
0.0 & 0.0 & 0.00426012 & 0.0 \\
0.04686132 & 0.00426012 & 0.00426012 & 0.0 \\
0.00426012 & 0.0 & 0.00426012 & 0.0 \\
0.0 & 0.0 & 0.00426012 & 0.38767092
\end{array}\right], \\
& \mathbf{D}_{5}=\left[\begin{array}{cccc}
0.0 & 0.0 & 0.00201216 & 0.0 \\
0.02213376 & 0.00201216 & 0.00201216 & 0.0 \\
0.00201216 & 0.0 & 0.00201216 & 0.0 \\
0.0 & 0.0 & 0.00201216 & 0.18310656
\end{array}\right],
\end{aligned}
$$

which gives $\bar{\pi}=[0.42982456,0.34586466,0.11027569,0.11403508]$ and $\lambda^{*}=0.70921066$.

Example 1 In this example, we choose ICT distribution as matrix exponential (ME) with representation $M E(\boldsymbol{\beta}, \mathbf{T}, \boldsymbol{\eta})$, see Gupta et al. [14] which covers a wide variety of distributions that arise in many real-life applications. Parameters are chosen as

$$
\boldsymbol{\beta}=\left[\begin{array}{lll}
2 \pi^{2}+1 & 0 & 0
\end{array}\right], \quad \mathbf{T}=\left[\begin{array}{ccc}
0 & 1 & 0 \\
0 & 0 & 1 \\
-2 \pi^{2}-1 & -2 \pi^{2}-4 & -4
\end{array}\right], \quad \boldsymbol{\eta}=\left[\begin{array}{l}
0 \\
0 \\
1
\end{array}\right]
$$

which gives $\mu=0.87362679$. Also we choose $p=0.6$, so that $\rho=0.541200326312$. One can also verify that $\rho=1-\pi_{0}^{+} \boldsymbol{e}$. Population size distribution at various epochs are shown in Table 3 and in the last row of table first two moments are also given at each epoch. For the numerical work, $\mathbf{A}(z)$ is obtained as (see, Gupta et al. [14])

$$
\mathbf{A}(z)=\left((\mathbf{I} \otimes \boldsymbol{\beta})(\mathbf{D}(z) \oplus \mathbf{T})^{-1}(\mathbf{I} \otimes-\boldsymbol{\eta})\right),
$$

and $\mathbf{S}(z)$ as

$$
\mathbf{S}(z)=\frac{1}{\boldsymbol{\beta} \mathbf{T}^{-2} \boldsymbol{\eta}}\left((\mathbf{I} \otimes \boldsymbol{\beta})(\mathbf{D}(z) \oplus \mathbf{T})^{-1}\left(\mathbf{I} \otimes \mathbf{T}^{-1} \boldsymbol{\eta}\right)\right)
$$

Remark 5 For Example-1, there are total 244 roots of the characteristic equation $\phi(z)=0$. Out of 244 roots, $4(=m)$ roots are in $|z| \leq 1$ and remaining roots lies outside the unit circle. In Figs. 1 and 2 , roots are shown in polar form. One important observation is that the roots 


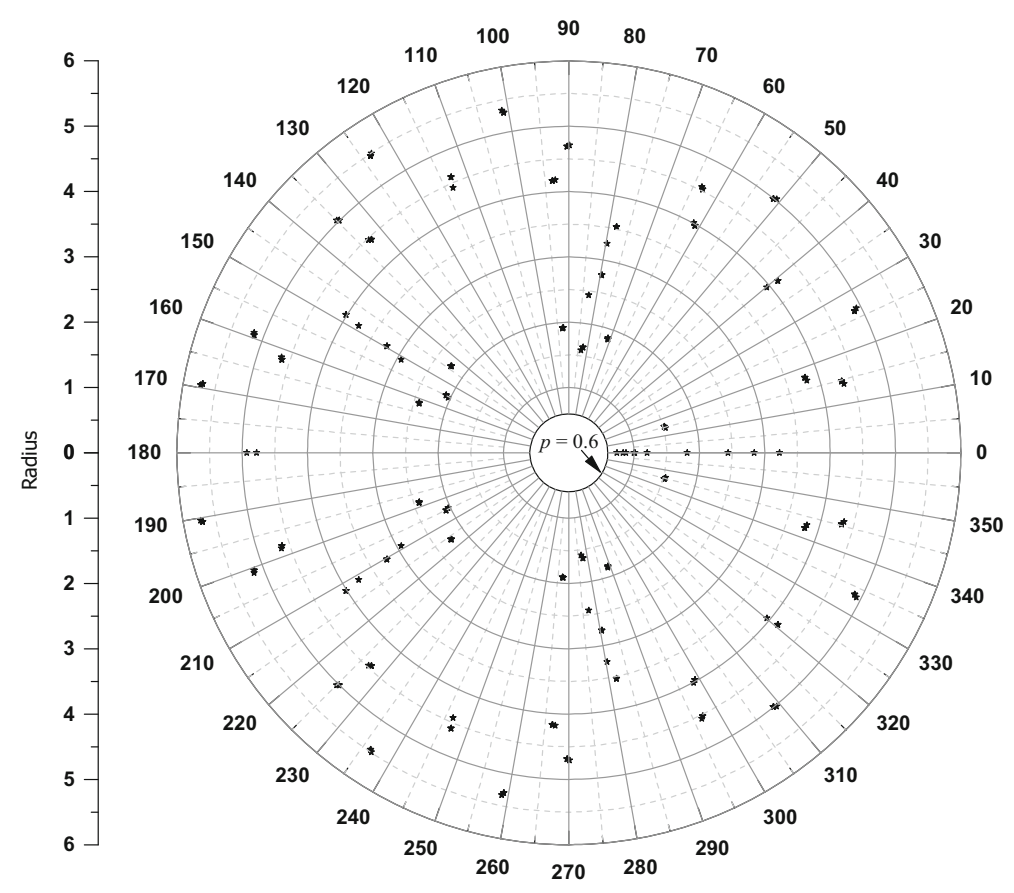

Fig. 1 All the roots of $\phi(z)=0$

inside the unit circle (i.e., 0.73816792, 0.82914014, 0.87674991 and 1.0) lies in $(p, 1]$, (see, Figs. 1-2). Since the VGF given in (1) is valid for $p<|z| \leq 1$, see Theorem 3.1 in [12, p216]. Similar pattern is also observed in upcoming examples 2 and 3 . Here, the inside roots are real but they may be complex for some other parameters. In Example 5-6, we are getting complex roots inside the unit circle, e.g., in Example-5, for $\lambda^{*}=1, p=0.5$, the inside roots are $0.952609-0.013606 i, 0.952609+0.013606 i, 1$.

Remark 6 During the numerical investigation we have performed three accuracy check (i) $\rho=1-\boldsymbol{\pi}_{0}^{+} \boldsymbol{e}$, (ii) $\overline{\boldsymbol{\pi}}=\boldsymbol{\Pi}^{+}$(1), (iii) factorial moments obtained from (23) are matched with the one obtained from Theorem-1 and 2 .

Example 2 In this example, we choose ICT distribution as phase-type (PH) distribution with representation $P H(\boldsymbol{\beta}, \mathbf{T})$. Parameters are chosen as

$$
\boldsymbol{\beta}=\left[\begin{array}{lll}
0.25 & 0.4 & 0.35
\end{array}\right], \quad \mathbf{T}=\left[\begin{array}{ccc}
-2.0 & 2.0 & 0.0 \\
0.0 & -2.0 & 2.0 \\
0.0 & 0.0 & -2.0
\end{array}\right]
$$

which gives $\mu=1.052632$, and we choose $p=0.6$ so $\rho=0.4491667\left(=1-\pi_{0}^{+} \boldsymbol{e}\right)$. Population size distribution at various epochs are given in Table 4 and in the last row of table first two moments are given. Inside roots are 0.77026917, 0.84787019, 0.89285998, 1.0, these lie in $(p, 1]$. In case of $\mathrm{PH}$ distribution, the expression of $\mathbf{A}(z)$ and $\mathbf{S}(z)$ can be obtained by setting $\boldsymbol{\eta}=-\mathbf{T} \boldsymbol{e}$ in (59)-(60) and is given as

$$
\mathbf{A}(z)=\left((\mathbf{I} \otimes \boldsymbol{\beta})(\mathbf{D}(z) \oplus \mathbf{T})^{-1}(\mathbf{I} \otimes \mathbf{T} \boldsymbol{e})\right),
$$




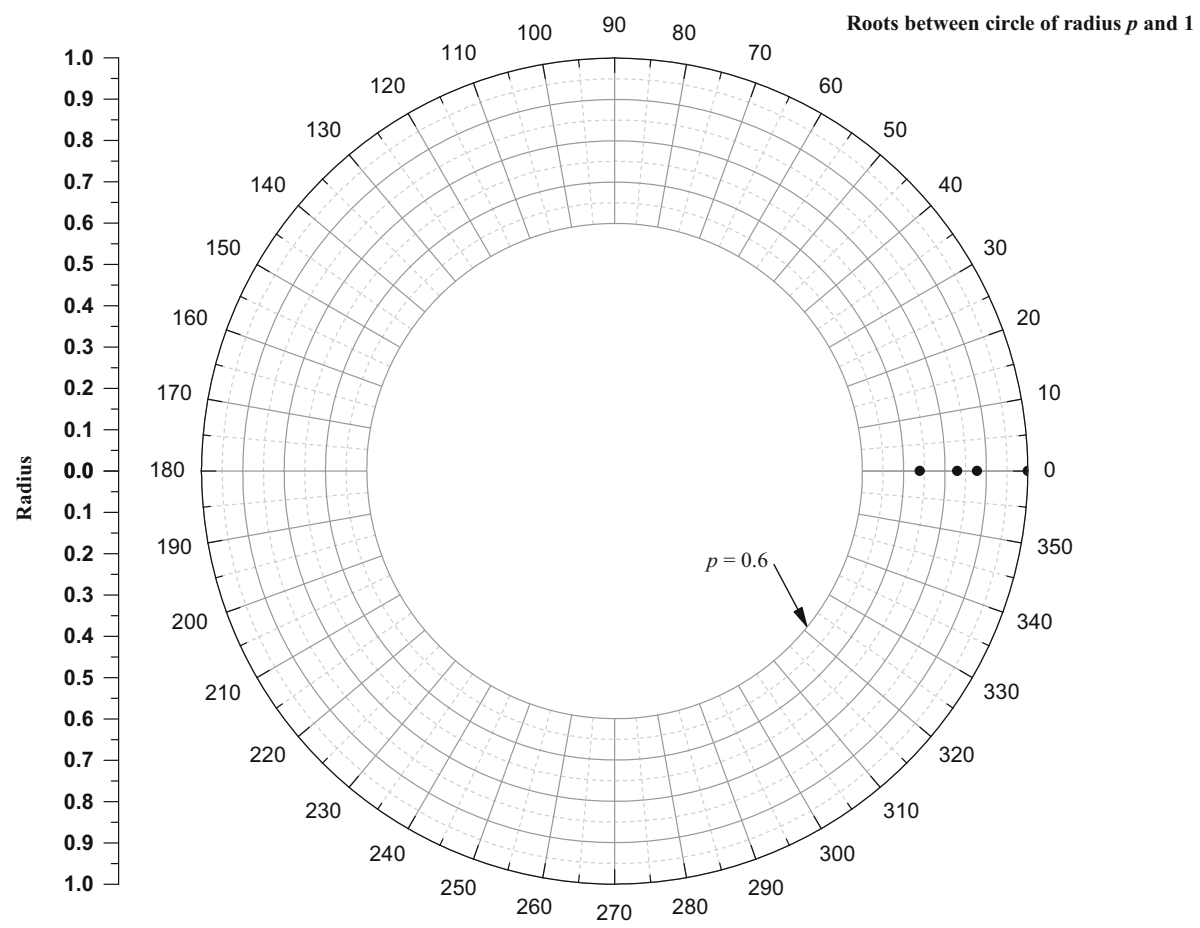

Fig. 2 Roots inside the unit circle

and

$$
\mathbf{S}(z)=\frac{1}{-\boldsymbol{\beta} \mathbf{T}^{-1} \boldsymbol{e}}\left((\mathbf{I} \otimes \boldsymbol{\beta})(\mathbf{D}(z) \oplus \mathbf{T})^{-1}(\mathbf{I} \otimes-\boldsymbol{e})\right) .
$$

The expression of $\mathbf{S}(z)$ given in (62) can also be found in [12, p228].

Example 3 In this example, we take ICT distribution as deterministic (D) with parameter $\mu=$ 1 and $p=0.6$, so $\rho=0.47280710\left(=1-\pi_{0}^{+} \boldsymbol{e}\right)$. Since the L.-S.T. of D is a transcendental function, so we have carried out the numerical analysis after rationalized it using Padé approximation, see Singh et al. [25,27]. Population size distribution at various epochs are shown in Table 5 and in the last row of table first two moments are given. Roots inside the unit circle are $0.71845781,0.83021863,0.88185547,1.0$, these lie in $(p, 1]$.

Now we analyze the effect of correlation (Corr) of batch arrival process, arrival rate $\left(\lambda^{*}\right)$, catastrophe arrival rate $(\mu)$, and ICT distributions on mean (1st moment) and 2nd moment for various values of $p$.

Example 4 In this example, we investigate the effect of correlation of the batch arrival on $m_{1}^{+}$ and $m_{2}^{+}$. We choose batch arrival process with negative, zero, and positive correlation with $\lambda^{*}=0.20168094$. They are given as

Negative Correlation We choose following set of matrices with Corr $=-0.488910$

$\mathbf{D}_{0}=\left[\begin{array}{ccc}-0.03181651 & 0.03181651 & 0.0 \\ 0.0 & -0.03181651 & 0.0 \\ 0.0 & 0.0 & -7.16666667\end{array}\right], \mathbf{D}_{i}=\frac{i^{2}}{204}\left[\begin{array}{ccc}0.0 & 0.0 & 0.0 \\ 0.00031809 & 0.0 & 0.03149841 \\ 7.09500000 & 0.0 & 0.07166667\end{array}\right], \quad(1 \leq$ $i \leq 8)$. 
1

స్ర

1 प ते ले $m$ के

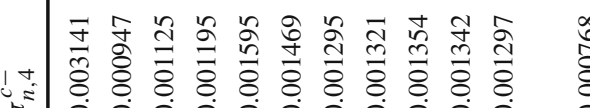

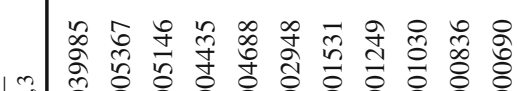

$\mu=1$ \%

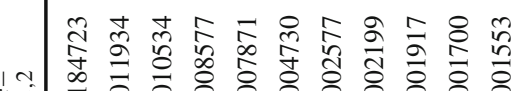

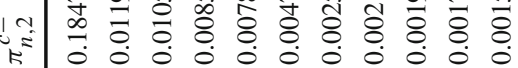

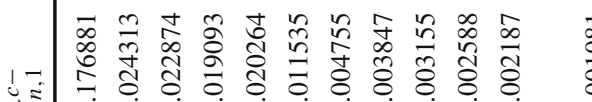

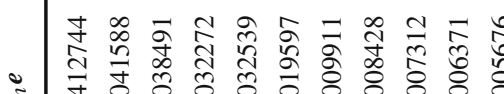

§ ఫ

8

ㅎํㅇ

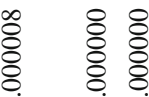

1

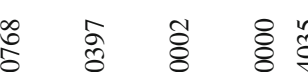

$\stackrel{8}{8}$

ฮิ

政

8

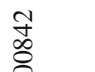

¿

$\circ$

$\stackrel{\infty}{\circ}$

हิे

กี

+

म

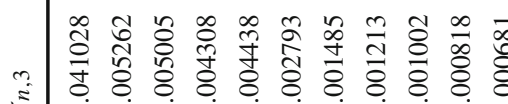

₹أl

U

글

䒽

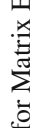

है

के

(2)

ส

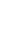

章

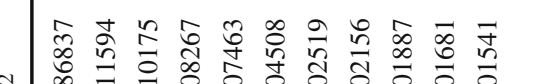

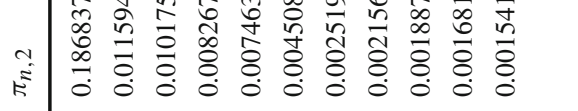

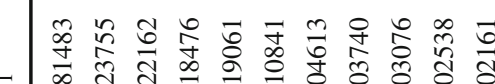

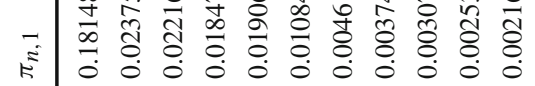

高

용

ले

$\stackrel{\infty}{5}$

ஓ.

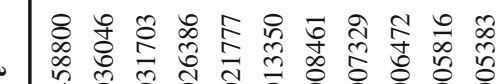

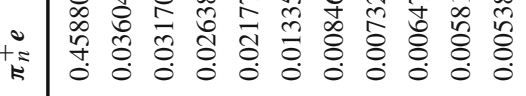

$\begin{array}{lllllllllll}0 & 0 & 0 & 0 & 0 & 0 & 0 & 0 & 0 & 0 & 0\end{array}$

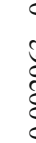

ते

ठิ.

- 0

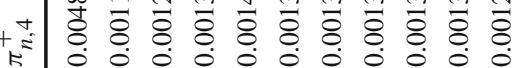

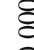

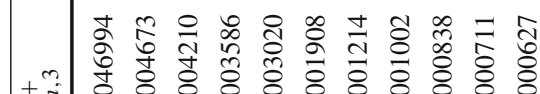

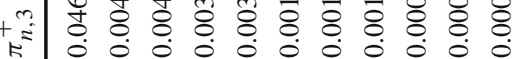

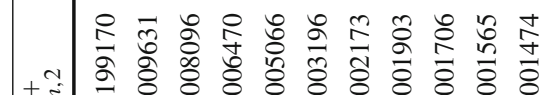

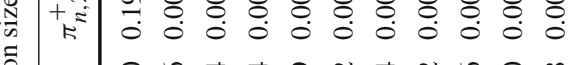

๙ै

ֻัญ

○.

กิ

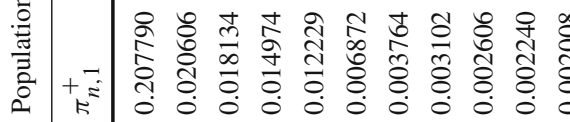

$\frac{m}{\frac{0}{0}}$

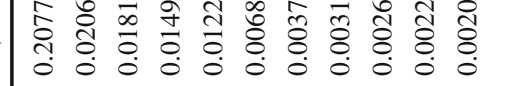

$\stackrel{8}{8}$

:

రิ

छे

‡ิ

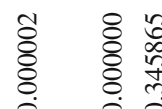

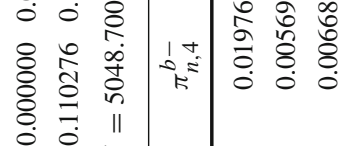

$\S$

$\stackrel{\infty}{8}$

ఠิ

$\frac{8}{8}$

8.

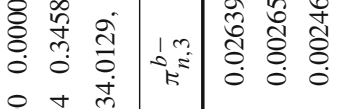

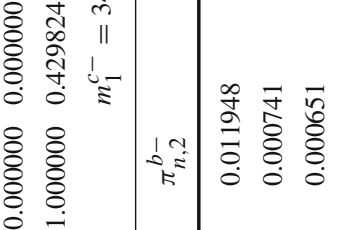

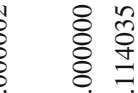

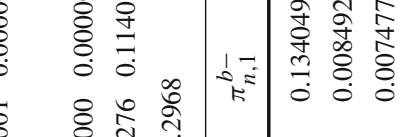

० :

官

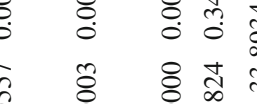

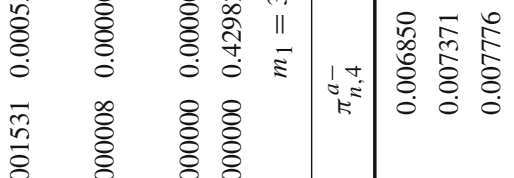

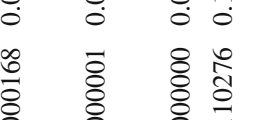

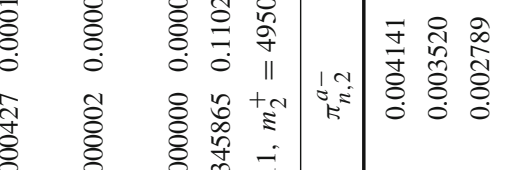

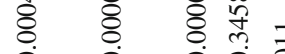

$\begin{array}{llll}0 & 0 & 0 & 0\end{array}$

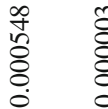

\&. 


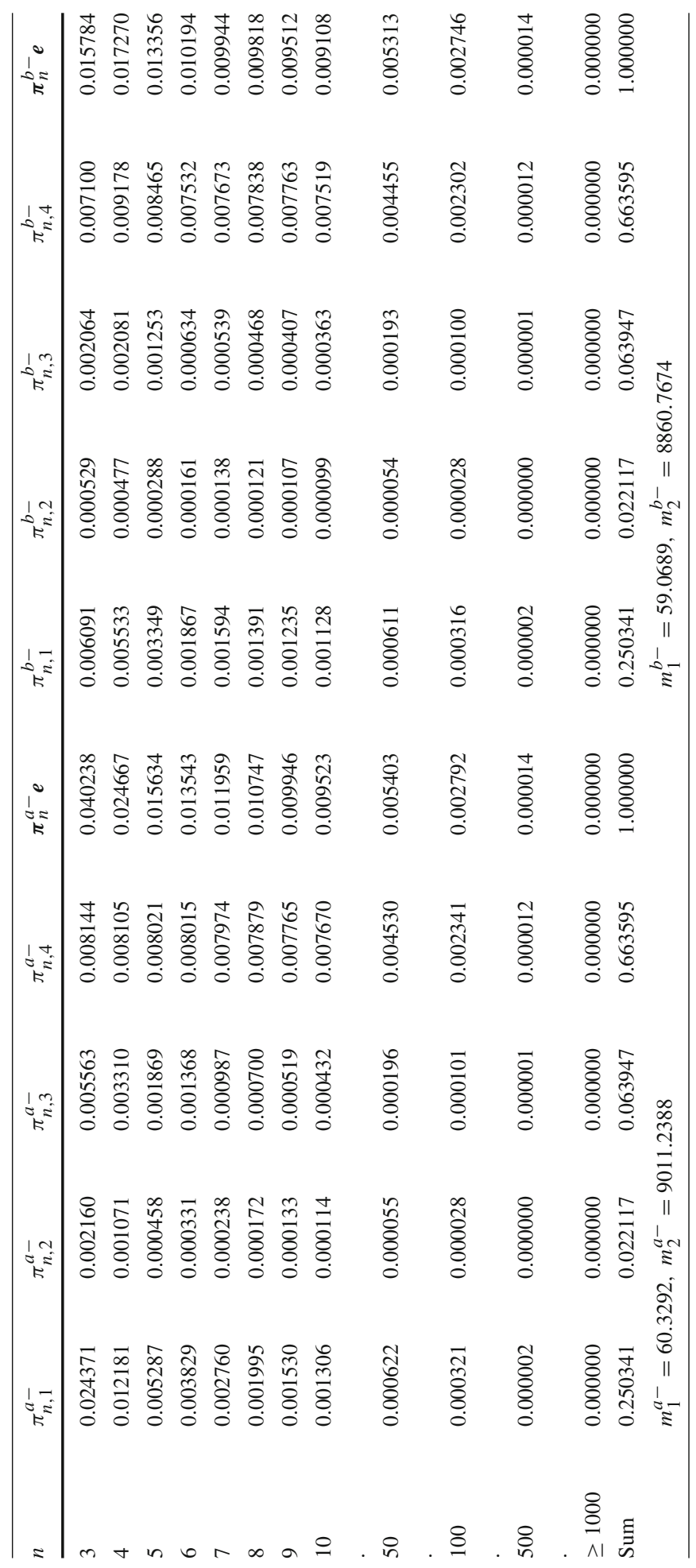




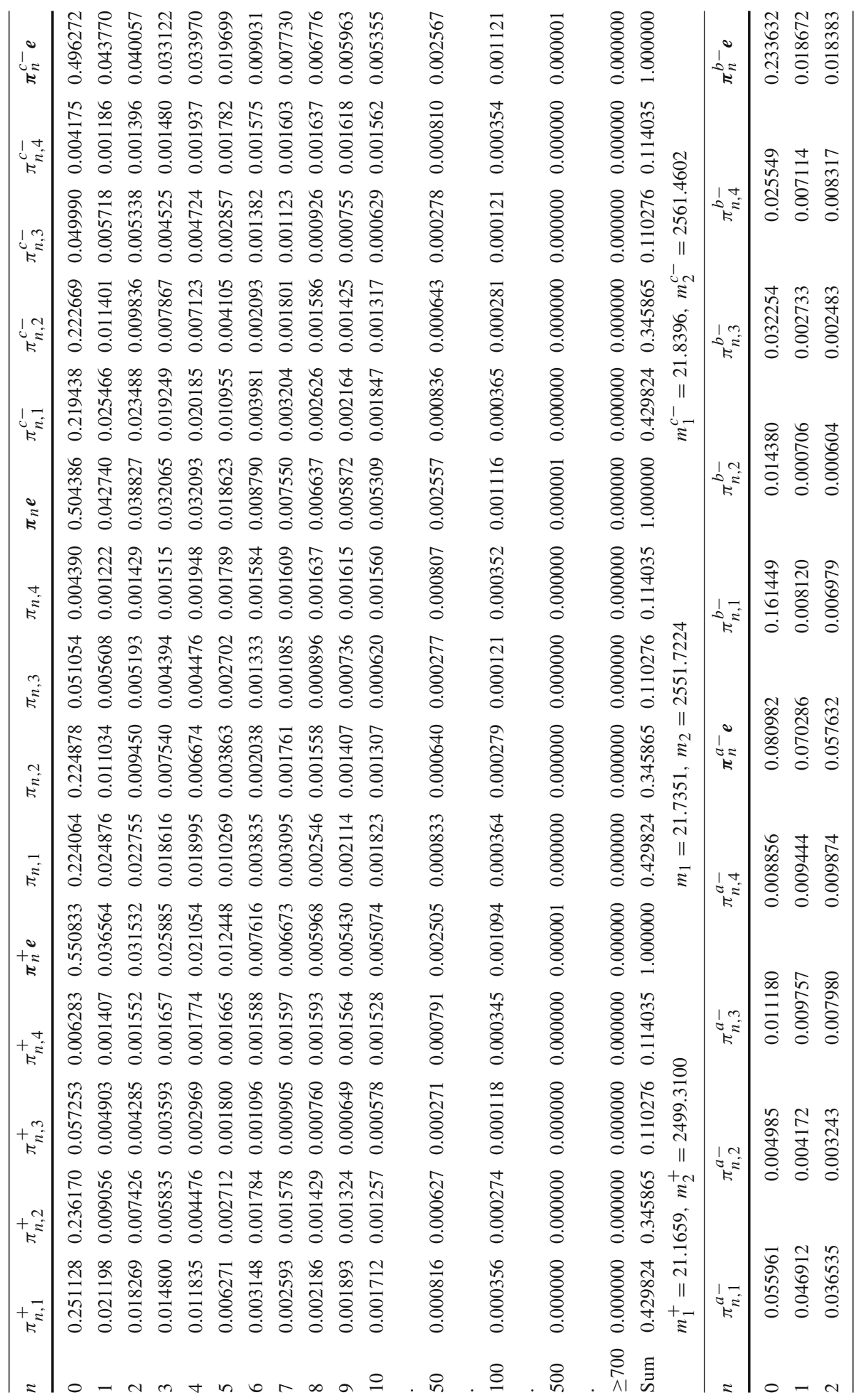




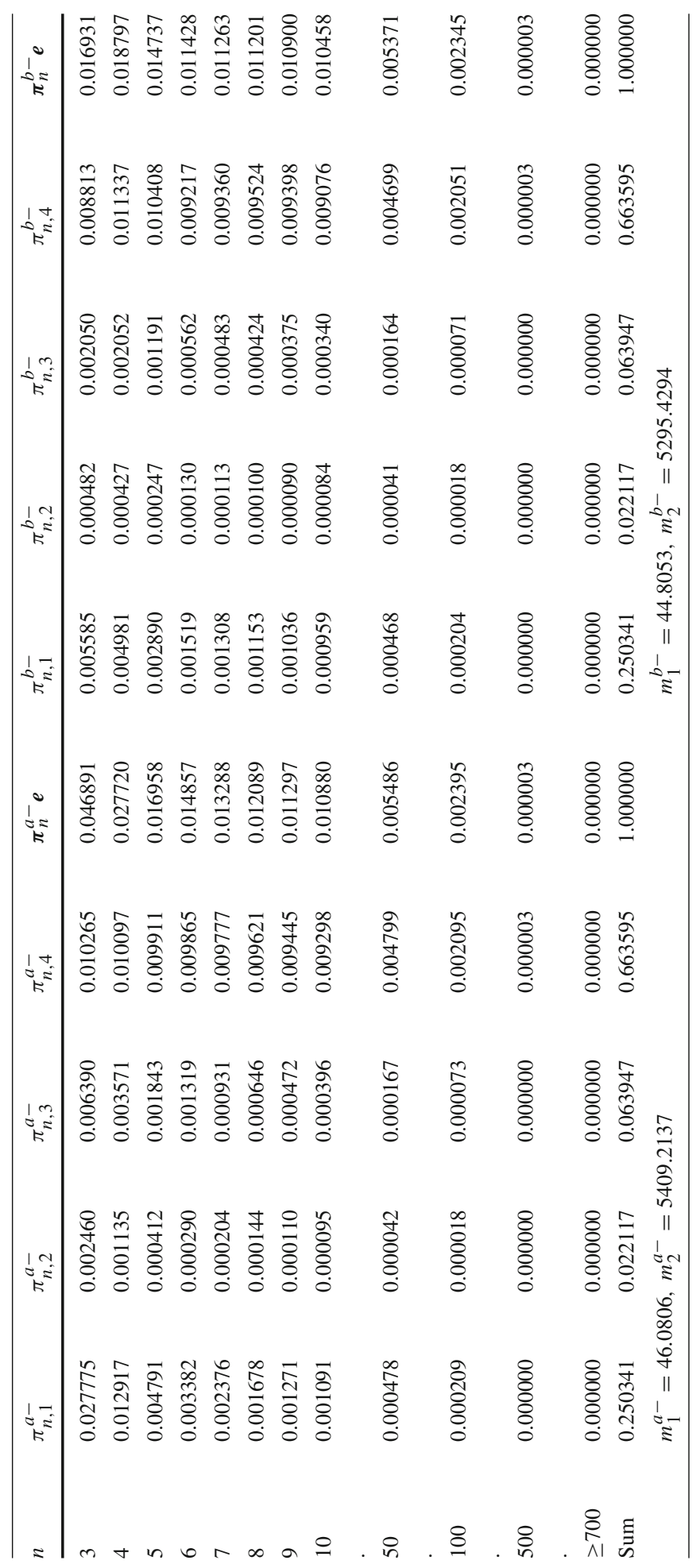




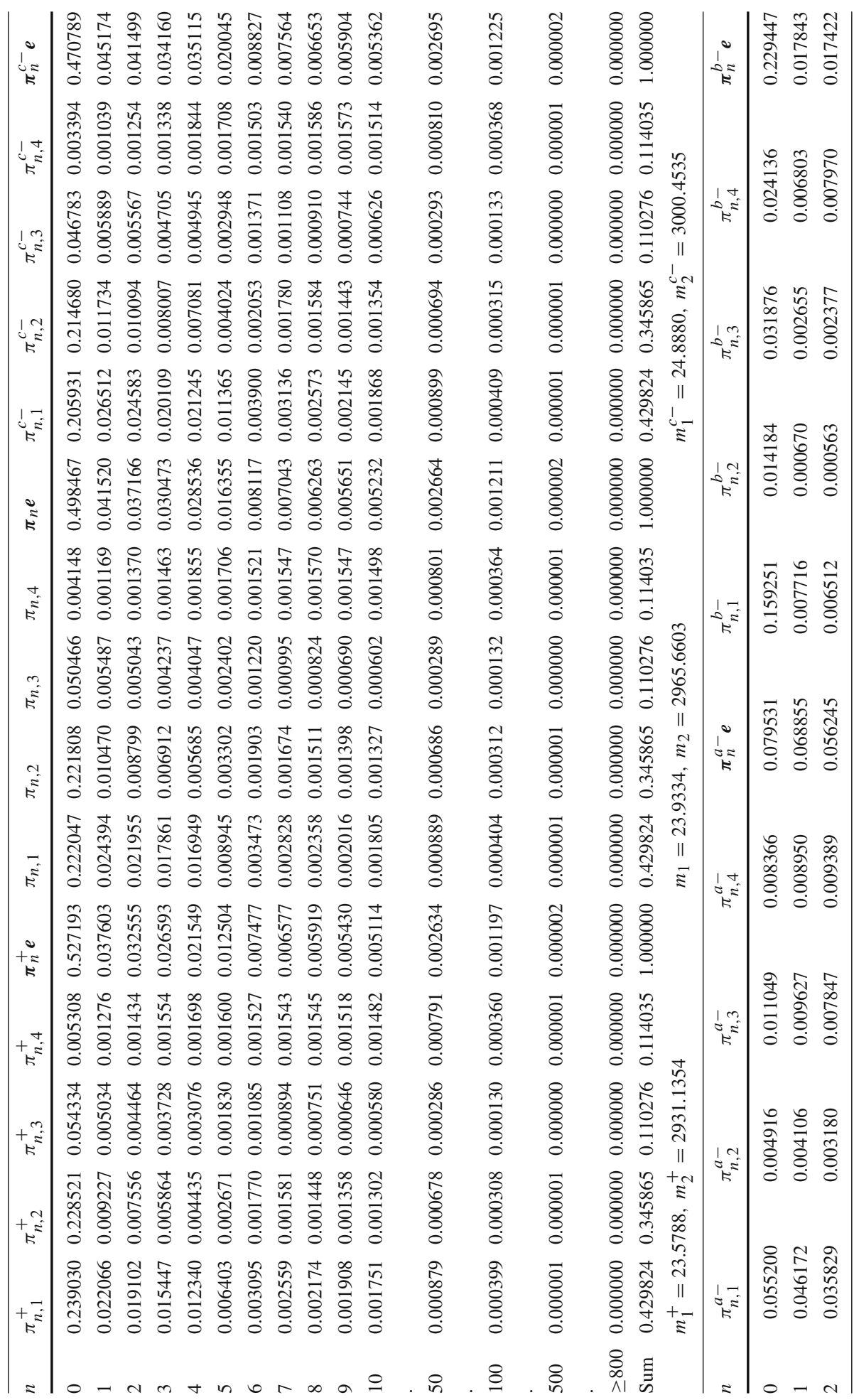




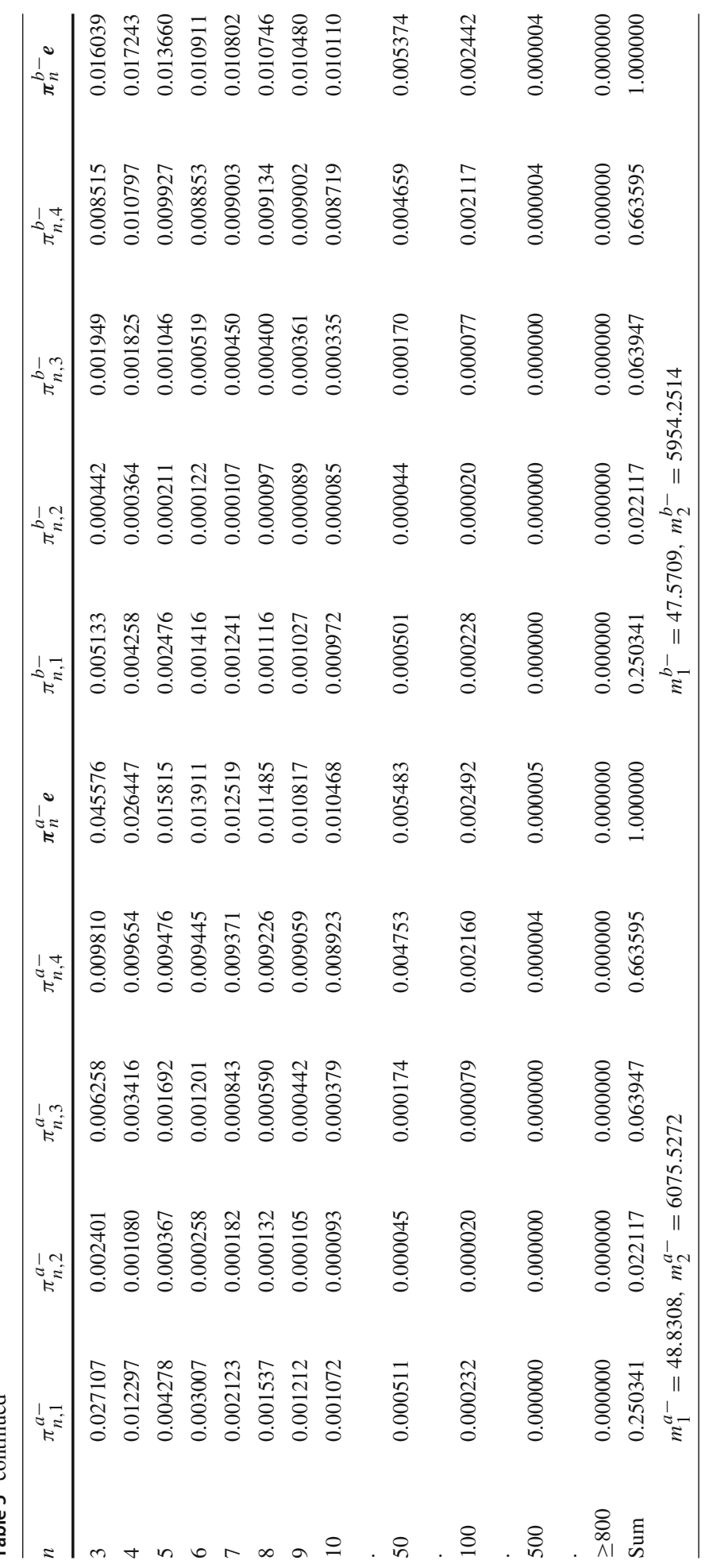




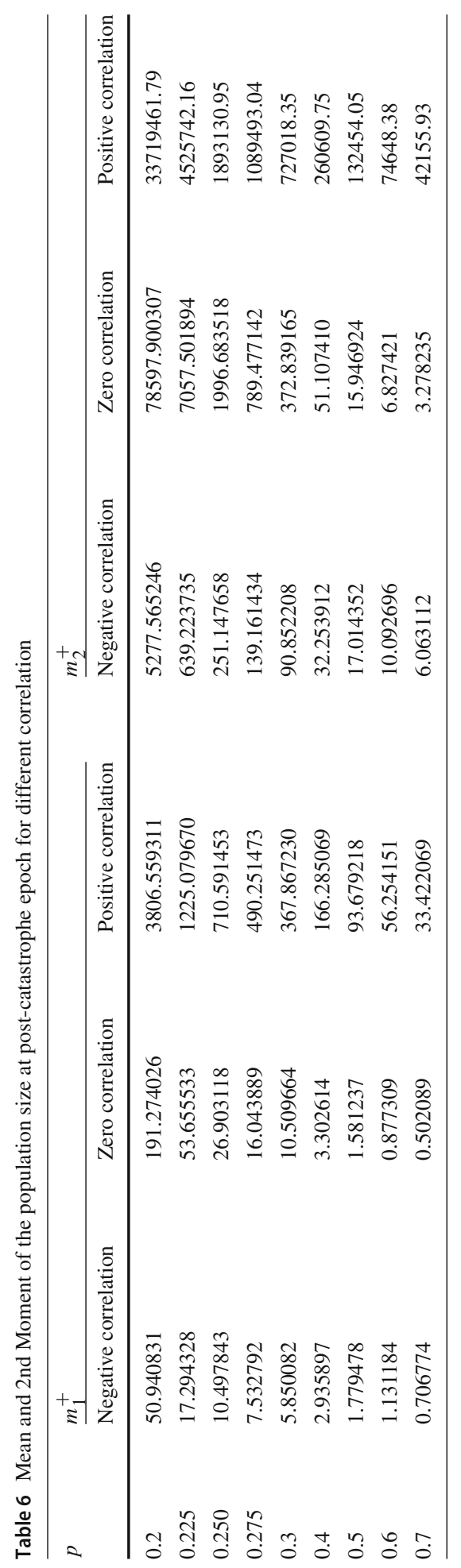


Fig. 3 Effect of correlation on mean for varying $p$

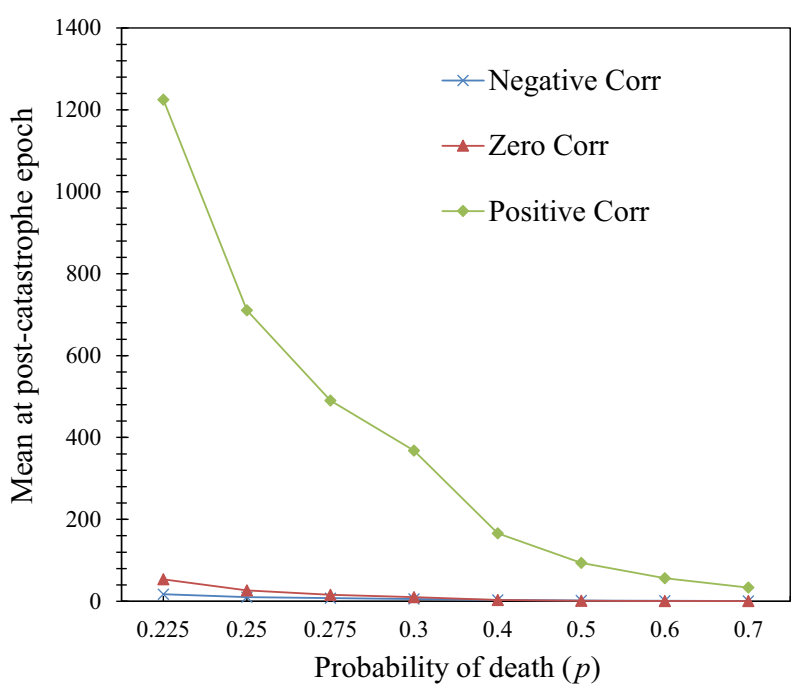

Zero Correlation We choose following set of matrices with Corr $=0.0$

$\mathbf{D}_{0}=\left[\begin{array}{cc}-0.06031754 & 0.0 \\ 0.0 & -0.00603175\end{array}\right], \mathbf{D}_{i}=\frac{i^{2}}{204}\left[\begin{array}{cc}0.05428579 & 0.00603175 \\ 0.00542858 & 0.00060317\end{array}\right], \quad(1 \leq i \leq 8)$.

Positive Correlation We choose following set of matrices with Corr $=0.488883$

$$
\mathbf{D}_{0}=\left[\begin{array}{ccc}
-0.03182002 & 0.03182002 & 0.0 \\
0.0 & -0.03182002 & 0.0 \\
0.0 & 0.0 & -7.16745777
\end{array}\right], \mathbf{D}_{i}=\frac{i^{2}}{204}\left[\begin{array}{ccc}
0.0 & 0.0 & 0.0 \\
0.03150189 & 0.0 & 0.00031813 \\
0.07167457 & 0.0 & 7.09578319
\end{array}\right], \quad(1 \leq
$$

ICT distribution is taken as ME and it is the same as the one given in Example-1 with $\mu=0.87362679$.

The numerical values are shown in Table 6 for various values of $p$. Moreover, the graphical analysis is displayed in Figs. 3, 4, and 5. We have presented the effect of different Corr on $m_{1}^{+}$in Fig. 3, and the effect of negative and zero Corr on $m_{1}^{+}$and $m_{2}^{+}$in Figs. 4 and 5 for various values of $p$. Since the values of $m_{1}^{+}$and $m_{2}^{+}$are sufficiently large for positive Corr as compared to negative and zero Corr, so the data is not shown in Figs. 4 and 5 for positive Corr.

One can observe the following from the Table 6 and Figs. 3, 4, 5.

1. For a fixed value of $p, m_{1}^{+}$and $m_{2}^{+}$are very large for positive Corr and very low for negative Corr. Since positive Corr results in the more number of arrivals in the system and hence it leads to increment of $m_{1}^{+}$and $m_{2}^{+}$.

2. For a fixed Corr, $m_{1}^{+}$and $m_{2}^{+}$decrease with the increment in $p$. Since increment in the value of $p$ results in large number of deaths in the system and hence it leads to the decrement of $m_{1}^{+}$and $m_{2}^{+}$.

3. There is significant variation in $m_{1}^{+}$and $m_{2}^{+}$for low values of $p$, e.g., $p \in(0.2,0.4)$ in comparison of large value of $p$. Since the low value of $p$ implies that the number of deaths will be very low which results in significant variation of $m_{1}^{+}$and $m_{2}^{+}$.

Example 5 In this example we analyze the effect of arrival rate for $\lambda^{*}=0.5,1.0,2.0,3.0$ on $m_{1}^{+}$and $m_{2}^{+}$for various values of $p$ which is displayed in Figs. 6 and 7 . We choose batch arrival process with the following matrix representation 


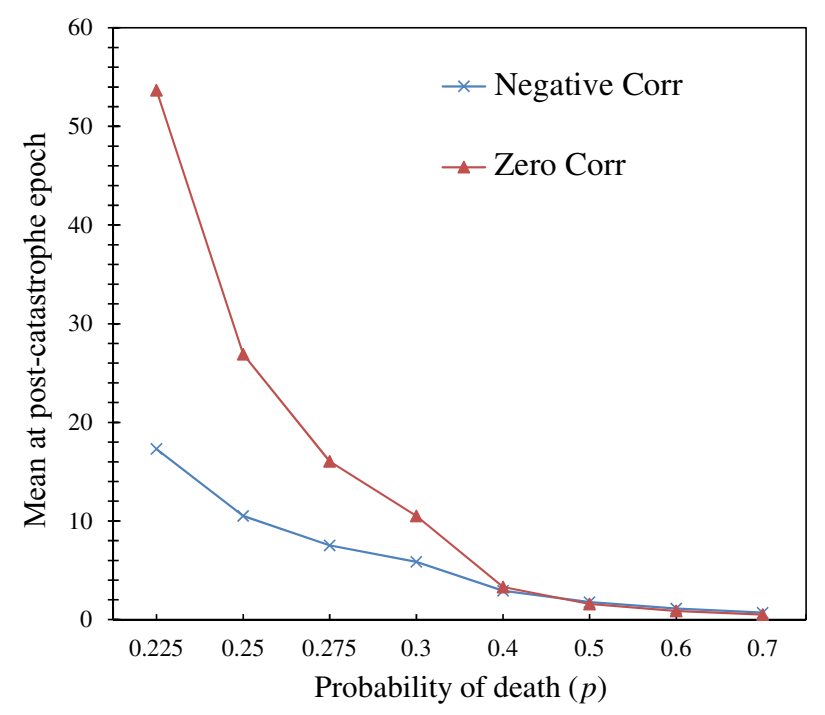

Fig. 4 Effect of negative and zero correlation on mean for varying $p$

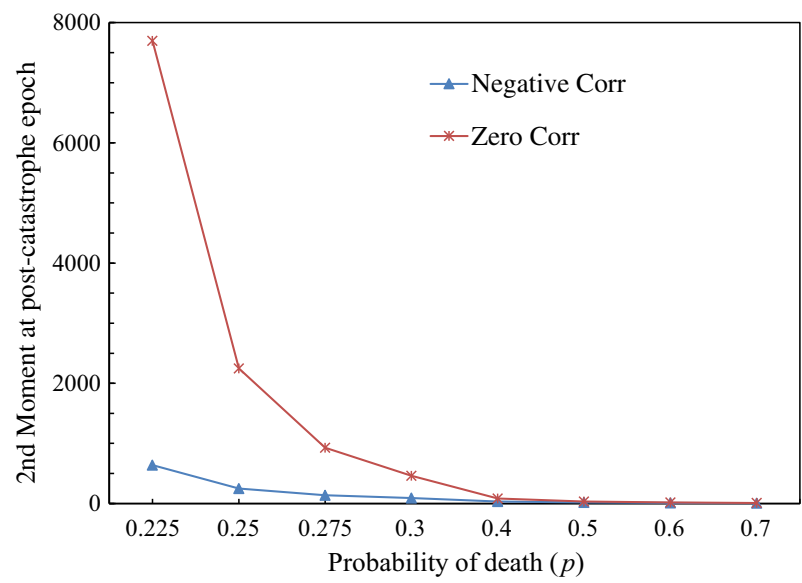

Fig. 5 Effect of negative and zero correlation on 2nd moment for varying $p$

$$
\begin{gathered}
\mathbf{D}_{0}=\sigma\left[\begin{array}{ccc}
-0.54190726 & 0.22817148 & 0.02852143 \\
0.04278215 & -0.61321085 & 0.42782152 \\
0.15686789 & 0.02852143 & -0.47060367
\end{array}\right], \\
\mathbf{D}_{i}=\frac{\sigma i^{2}}{55}\left[\begin{array}{lll}
0.08556430 & 0.12834646 & 0.07130359 \\
0.02852143 & 0.09982502 & 0.01426072 \\
0.11408574 & 0.07130358 & 0.09982502
\end{array}\right],(1 \leq i \leq 5), \text { which gives } \lambda^{*}=\sigma .
\end{gathered}
$$

Here ICT distribution is chosen as PH with $\boldsymbol{\beta}=\left[\begin{array}{lll}0.5 & 0.5 & 0.0\end{array}\right]$ and $\mathbf{T}=\left[\begin{array}{ccc}-33.0 & 33.0 & 0.0 \\ 0.0 & -33.0 & 33.0 \\ 0.0 & 0.0 & -33.0\end{array}\right]$ which gives $\mu=13.20$.

One can observe the following from the Figs. 6 and 7. 


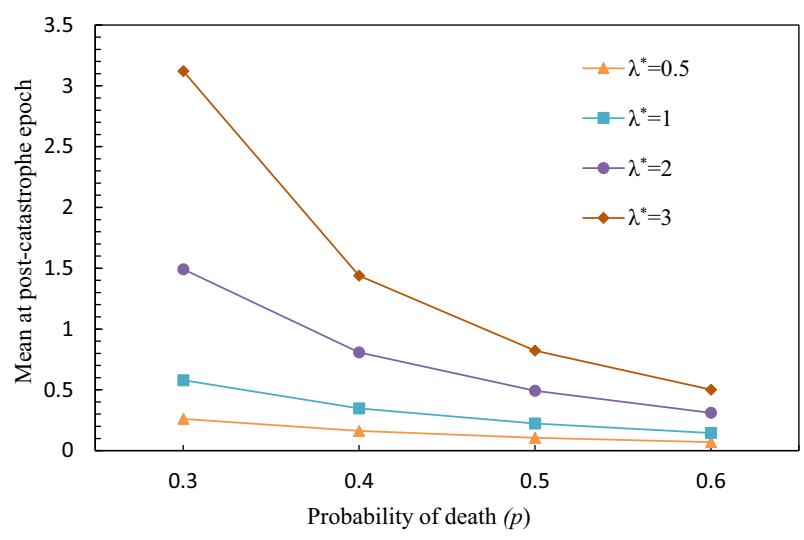

Fig. 6 Effect of arrival rate on Mean for varying $p$

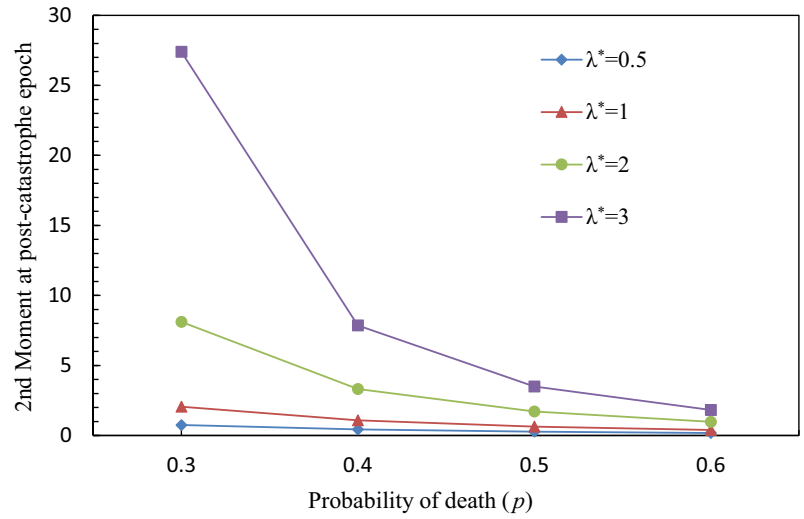

Fig. 7 Effect of arrival rate on 2nd Moment for varying $p$

1. For a fixed value of $p, m_{1}^{+}$and $m_{2}^{+}$are in increasing order as $\lambda^{*}$ increases. Since the increment in $\lambda^{*}$ results in the more number of arrivals into the system which results in the increment of $m_{1}^{+}$and $m_{2}^{+}$.

2. For a fixed $\lambda^{*}, m_{1}^{+}$and $m_{2}^{+}$decrease with the increment in $p$. Since the increment in the value of $p$ results in large number of deaths in the system which leads to the decrement of $m_{1}^{+}$and $m_{2}^{+}$.

3. There is significant variation in $m_{1}^{+}$and $m_{2}^{+}$for low values of $p$ (see $p \leq 0.3$ ). However, a small variation is observed for large value of $p$. Since a low value of $p$ implies that the number of deaths will be very less which results in significant variation of $m_{1}^{+}$and $m_{2}^{+}$. As $p$ tends to 1 the effect of $\lambda^{*}$ becomes almost negligible which is the case of total catastrophe, i.e., after the occurrence of catastrophe the population size will be zero (becomes extinct).

Example 6 In this example we analyze the effect of catastrophe arrival rate for $\mu=$ 1.0, 2.0, 3.0 on $m_{1}^{+}$and $m_{2}^{+}$for various values of $p$, see, Figs. 8 and 9. We choose batch arrival process with the following matrix representation 


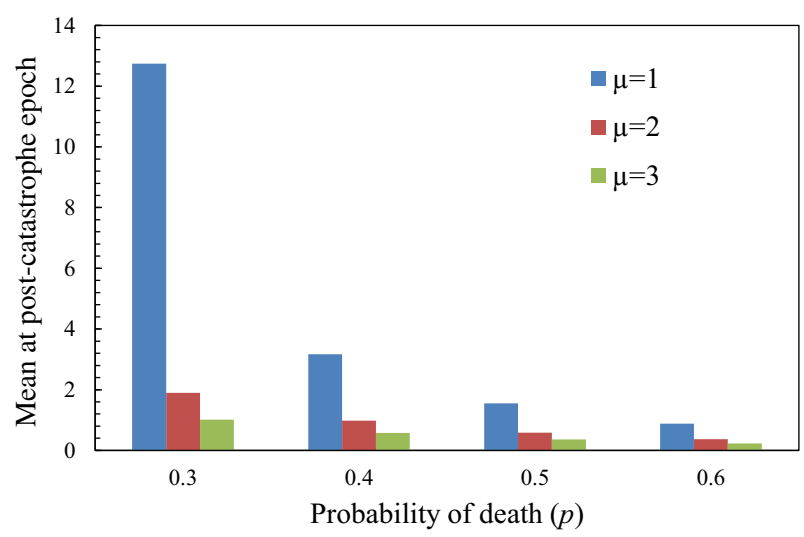

Fig. 8 Effect of catastrophe arrival rate $(\mu)$ on Mean for varying $p$

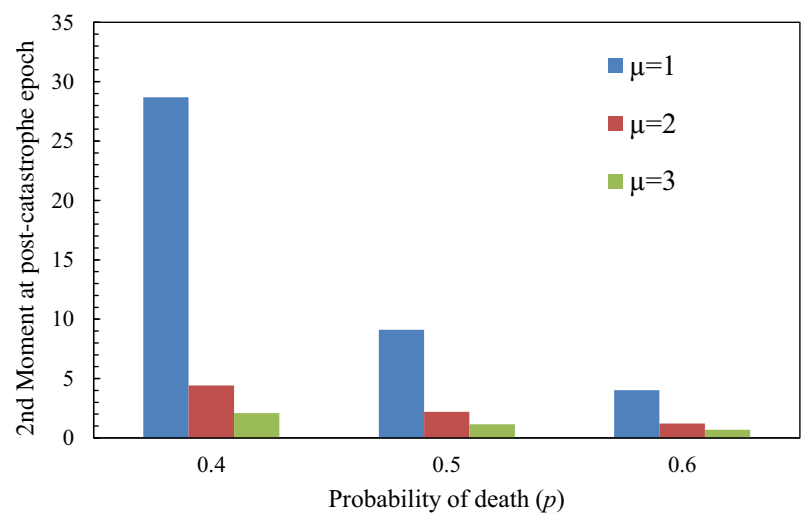

Fig. 9 Effect of catastrophe arrival rate $(\mu)$ on 2nd Moment for varying $p$

$\mathbf{D}_{0}=\left[\begin{array}{ccc}-0.1900 & 0.0800 & 0.0100 \\ 0.0150 & -0.2150 & 0.1500 \\ 0.0550 & 0.0100 & -0.1650\end{array}\right], \mathbf{D}_{i}=\frac{i^{2}}{55}\left[\begin{array}{ccc}0.0300 & 0.0450 & 0.0250 \\ 0.0100 & 0.0350 & 0.0050 \\ 0.0400 & 0.0250 & 0.0350\end{array}\right],(1 \leq i \leq 5)$, which gives $\lambda^{*}=0.3506135$. The ICT distribution is chosen as PH with $\boldsymbol{\beta}=\left[\begin{array}{lll}0.5 & 0.5 & 0.0\end{array}\right]$ and $\mathbf{T}=$ $\sigma\left[\begin{array}{ccc}-2.5 & 2.5 & 0.0 \\ 0.0 & -2.5 & 2.5 \\ 0.0 & 0.0 & -2.5\end{array}\right]$ which gives $\mu=\sigma$.

One can observe the following from the Figs. 8 and 9.

1. For a fixed value of $p$ both $m_{1}^{+}$and $m_{2}^{+}$are in decreasing order with the increment of $\mu$. Since increment in $\mu$ results in the more frequent occurrence of catastrophes which results in large number of deaths in the system and hence in the decrement of $m_{1}^{+}$and $m_{2}^{+}$.

2. For a fixed $\mu, m_{1}^{+}$and $m_{2}^{+}$decrease with the increment in $p$. This is due to the same reason as that of Example-5 point-2.

3. A very small variation is observed in $m_{1}^{+}$and $m_{2}^{+}$for large value of $p$. Since the high value of $p$ implies large number of deaths and then $\mu$ does not have much effect on $m_{1}^{+}$ and $m_{2}^{+}$. 


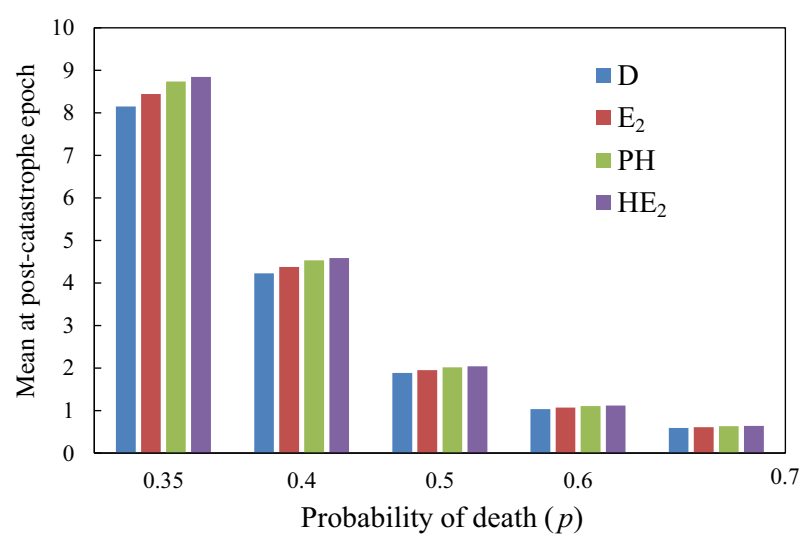

Fig. 10 Effect of ICT distributions on Mean for varying $p$

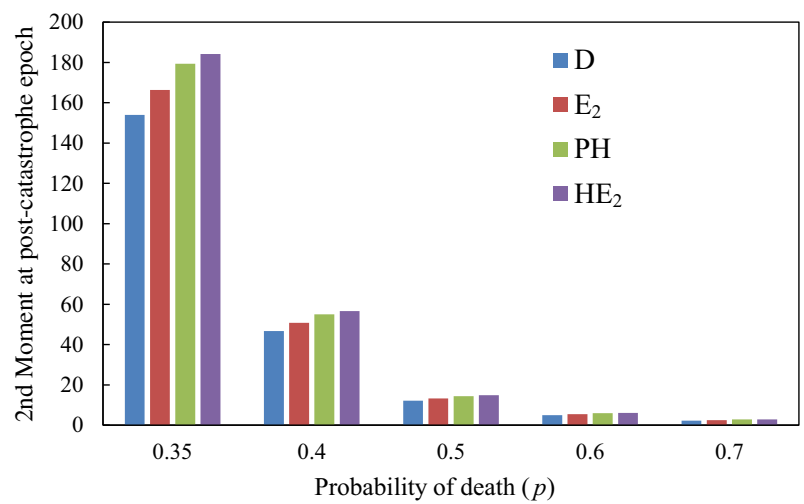

Fig. 11 Effect of ICT distributions on 2nd Moment for varying $p$

Example 7 In this example we analyze the effect of ICT distributions on $m_{1}^{+}$and $m_{2}^{+}$for various values of $p$, see, Figs. 10 and 11 . We have chosen batch arrival process as given in Example 6. Here the following ICT distributions are chosen with $\mu=0.87362679$.

Hyper-exponential $\left(H E_{2}\right): \boldsymbol{\beta}=\left[\begin{array}{ll}0.9 & 0.1\end{array}\right]$ and $\mathbf{T}=\left[\begin{array}{cc}-0.97069643 & 0.0 \\ 0.0 & -0.45980357\end{array}\right]$ with $\mathrm{CV}=1.086278$.

Phase-type $(P H): \quad \beta=\left[\begin{array}{ll}0.9 & 0.1\end{array}\right]$ and $\mathbf{T}=\left[\begin{array}{cc}-1.74725358 & 0.87362679 \\ 0.0 & -0.87362679\end{array}\right]$ with $\mathrm{CV}=1.0$

Erlang $\left(E_{2}\right): \boldsymbol{\beta}=\left[\begin{array}{ll}1.0 & 0.0\end{array}\right]$ and $\mathbf{T}=\left[\begin{array}{cc}-1.74725358 & 1.74725358 \\ 0.0 & -1.74725358\end{array}\right]$ with $\mathrm{CV}=0.707107$.

Deterministic (D) With $\mathrm{CV}=0$.

Where CV stands for coefficient of variation, and is given by $C V=\sqrt{\text { Variance }} /$ Mean .

One can observe the following from the Figs. 10-11.

1. For a fixed value of $p, m_{1}^{+}$and $m_{2}^{+}$follow the pattern as $D<E_{2}<P H<H E_{2}$, this is due to the CV of the distribution. Since CV of distributions are 0.0 (for $D)<$ 0.707107 (for $\left.E_{2}\right)<1$ (for $P H$ ) $<1.086278$ (for $H E_{2}$ ).

2. For a fixed ICT distribution, $m_{1}^{+}$and $m_{2}^{+}$decrease with the increment in $p$. It is due to the same reason as that of Example-5, point-2. 
3. Less variation is observed in $m_{1}^{+}$and $m_{2}^{+}$for a large value of $p$. This is due to the same reason as that of Example-6 point-3.

Remark 7 From the analysis of Examples 4-7, one can conclude that increment in Corr, $\lambda^{*}$, or CV of ICT distribution results in the increment of $m_{1}^{+}$and $m_{2}^{+}$whereas increment in $p$ or $\mu$ results in the decrement of $m_{1}^{+}$and $m_{2}^{+}$.

\section{Conclusion}

In this paper, we have developed an entirely different procedure, which is based on the roots of the characteristic equation, to analyze the population model that grows according to batch Markovian arrival process and is subjected to renewal generated geometric catastrophes. The explicit expression of population size distributions at various epochs are given in terms of the roots. The whole methodology is demonstrated by considering several numerical examples. The effect of various model parameters on the mean population size and the second moment is also critically examined, and relevant conclusions have been drawn. It is observed that each parameter has its own importance, however, the effect of $p$ as well as the correlation between the inter-arrival times of the batches play a major role. We finally hope that the results presented in this paper would be useful to the researchers and practitioners involved in a study of the effect of catastrophes such as Covid-19 on the population growth model.

Acknowledgements The first author Nitin Kumar is thankful to Indian Institute of Technology Kharagpur, India for the financial support. The authors would like to thank the editor and two anonymous referees for their valuable remarks and suggestions which led to the paper in current form.

Author Contributions NK analyzed and carried out the complete theoretical and numerical investigation of the model. UCG provided the idea to generate and solve the problem, and was a major contributor in writing the manuscript as well. GS given the idea to handle the roots of the characteristic equation, and also provided some graphical results. All authors read and approved the final manuscript.

Funding Not applicable

\section{Declarations}

Conflict of interest The authors have no conflicts of interest to declare.

Availability of data and material Not applicable

Code availability Not applicable

\section{References}

1. Ahmad, I., Khan, M.N., Inc, M., Ahmad, H., Nisar, K.S.: Numerical simulation of simulate an anomalous solute transport model via local meshless method. Alex. Eng. J. 59(4), 2827-2838 (2020)

2. Ahmed, N., Rafiq, M., Baleanu, D., Rehman, MAu., Khan, I., Ali, M., Nisar, K.S.: Structure preserving algorithms for mathematical model of auto-catalytic glycolysis chemical reaction and numerical simulations. Eur. Phys. J. Plus 135(6), 1-21 (2020)

3. Ali, I., Haq, S., Nisar, K.S., Baleanu, D.: An efficient numerical scheme based on Lucas polynomials for the study of multidimensional Burgers-type equations. Adv. Differ. Equ. 1, 1-24 (2021)

4. Bank, B., Samanta, SK.: Analytical and computational studies of the $B M A P / G^{(a, Y)} / 1$ queue. Communications in Statistics-Theory and Methods pp 1-29 (2020) 
5. Barbhuiya, F.P., Kumar, N., Gupta, U.C.: Batch renewal arrival process subject to geometric catastrophes. Methodol. Comput. Appl. Probab. 21(1), 69-83 (2019)

6. Botta, R.F., Harris, C.M., Marchal, W.G.: Characterizations of generalized hyperexponential distribution functions. Stoch. Model. 3(1), 115-148 (1987)

7. Brockwell, P.J., Gani, J., Resnick, S.I.: Birth, immigration and catastrophe processes. Adv. Appl. Probab. 14(4), 709-731 (1982)

8. Chaudhry, M.L., Singh, G., Gupta, U.C.: A simple and complete computational analysis of $M A P / R / 1$ queue using roots. Methodol. Comput. Appl. Probab. 15(3), 563-582 (2013)

9. Economou, A.: On the control of a compound immigration process through total catastrophes. Eur. J. Oper. Res. 147(3), 522-529 (2003)

10. Economou, A.: The compound Poisson immigration process subject to binomial catastrophes. J. Appl. Probab. 41(2), 508-523 (2004)

11. Economou, A., Fakinos, D.: A continuous-time Markov chain under the influence of a regulating point process and applications in stochastic models with catastrophes. Eur. J. Oper. Res. 149(3), 625-640 (2003)

12. Economou, A., Gómez-Corral, A.: The batch Markovian arrival process subject to renewal generated geometric catastrophes. Stoch. Model. 23(2), 211-233 (2007)

13. Ghaffar, A., Ali, A., Ahmed, S., Akram, S., Baleanu, D., Nisar, K.S., et al.: A novel analytical technique to obtain the solitary solutions for nonlinear evolution equation of fractional order. Adv. Differ. Equ. 1, $1-15$ (2020)

14. Gupta, U.C., Singh, G., Chaudhry, M.L.: An alternative method for computing system-length distributions of $B M A P / R / 1$ and $B M A P / D / 1$ queues using roots. Perform. Eval. 95, 60-79 (2016)

15. Gupta, U.C., Kumar, N., Barbhuiya, F.P.: A queueing system with batch renewal input and negative arrivals. In: Joshua, V., Varadhan, S., Vishnevsky, V. (eds.) Applied Probability and Stochastic Processes, pp. 143-157. Infosys Science Foundation Series, Springer, Singapore (2020)

16. Jhangeer, A., Hussain, A., Junaid-U-Rehman, M., Khan, I., Baleanu, D., Nisar, KS.: Lie analysis, conservation laws and travelling wave structures of nonlinear Bogoyavlenskii-Kadomtsev-Petviashvili equation. Results in Physics 19:103492 (2020)

17. Khalid, A., Ghaffar, A., Naeem, M.N., Nisar, K.S., Baleanu, D.: Solutions of BVPs arising in hydrodynamic and magnetohydro-dynamic stability theory using polynomial and non-polynomial splines. Alex. Eng. J. 60(1), 941-953 (2021)

18. Kumar, N., Gupta, UC.: Analysis of a population model with batch Markovian arrivals influenced by Markov arrival geometric catastrophes. Communications in Statistics-Theory and Methods pp 1-22 (2019)

19. Kumar, N., Gupta, U.C.: Analysis of batch Bernoulli process subject to discrete-time renewal generated binomial catastrophes. Ann. Oper. Res. 287(1), 257-283 (2020a)

20. Kumar, N., Gupta, U.C.: A renewal generated geometric catastrophe model with discrete-time Markovian arrival process. Methodol. Comput. Appl. Probab. 22(3), 1293-1324 (2020b)

21. Kumar, N., Barbhuiya, F.P., Gupta, U.C.: Analysis of a geometric catastrophe model with discrete-time batch renewal arrival process. RAIRO Oper. Res. 54(5), 1249-1268 (2020a)

22. Kumar, N., Barbhuiya, F.P., Gupta, U.C.: Unified killing mechanism in a single server queue with renewal input. Opsearch 57(1), 246-259 (2020b)

23. Pradhan, S., Gupta, U.C.: Analysis of an infinite-buffer batch-size-dependent service queue with Markovian arrival process. Ann. Oper. Res. 277(2), 161-196 (2019)

24. Shaikh, A., Tassaddiq, A., Nisar, K.S., Baleanu, D.: Analysis of differential equations involving CaputoFabrizio fractional operator and its applications to reaction-diffusion equations. Adv. Differ. Equ. 1, 1-14 (2019)

25. Singh, G., Chaudhry, M.L., Gupta, U.C.: Computing system-time and system-length distributions for $M A P / D / 1$ queue using distributional Littles law. Perform. Eval. 69(2), 102-118 (2012)

26. Singh, G., Gupta, U.C., Chaudhry, M.L.: Computational analysis of bulk service queue with Markovian arrival process: $M A P / R^{(a, b)} / 1$ queue. Opsearch 50(4), 582-603 (2013)

27. Singh, G., Gupta, U.C., Chaudhry, M.L.: Analysis of queueing-time distributions for $M A P / D_{N} / 1$ queue. Int. J. Comput. Math. 91(9), 1911-1930 (2014)

28. Singh, G., Gupta, U.C., Chaudhry, M.L.: Detailed computational analysis of queueing-time distributions of the $B M A P / G / 1$ queue using roots. J. Appl. Probab. 53(4), 1078-1097 (2016)

Publisher's Note Springer Nature remains neutral with regard to jurisdictional claims in published maps and institutional affiliations. 